L. Giannone, J. Baldzuhn, R. Burhenn, P. Grigull,

U. Stroth, F. Wagner,

R. Brakel, C. Fuchs, H.-J. Hartfuss, K. McCormick, A.

Weller, C. Wendland

NBI Team, ECRH Team and the W7-AS Team

K. Itoh, S.-I. Itoh

Physics of the Density Limit in the W7-AS Stellarator

IPP III/252

Februar 2000 
L. Giannone, J. Baldzuhn, R. Burhenn, P. Grigull,

U. Stroth, F. Wagner,

R. Brakel, C. Fuchs, H.-J. Hartfuss, K. McCormick, A.

Weller, C. Wendland

NBI Team, ECRH Team and the W7-AS Team

K. Itoh, S.-I. Itoh

Physics of the Density Limit in the W7-AS Stellarator

IPP III/252

Februar 2000 
"Dieser IPP-Bericht ist als Manuskript des Autors gedruckt. Die Arbeit entstand im Rahmen der Zusammenarbeit zwischen dem IPP und EURATOM auf dem Gebiet der Plasmaphysik. Alle Rechte vorbehalten."

"This IPP-Report has been printed as author's manuscript elaborated under the collaboration between the IPP and EURATOM on the field of plasma physics. All rights reserved." 


\title{
Physics of the density limit in the W7-AS Stellarator
}

\author{
L. Giannone, J. Baldzuhn, R. Burhenn, P. Grigull, U. Stroth, F. Wagner, \\ R. Brakel, C. Fuchs, H.J. Hartfuss, K. McCormick, A. Weller, C. Wendland \\ NBI Team, ECRH Team and the W7-AS Team \\ Max Planck Institut für Plasmaphysik, \\ EURATOM-IPP Association, D-85748 Garching, FRG
}

\author{
K. Itoh, \\ National Institute for Fusion Science, \\ Toki 509-5292, Japan.
}

\begin{abstract}
S.-I. Itoh,
Research Institute for Applied Mechanics, Kyushu University, Kasuga, 816-8580, Japan.
\end{abstract}

March 8, 2000

\begin{abstract}
Density limit discharges in the W7-AS stellarator, with constant line integrated density and a duration of up to 2 seconds, were studied at three values of the toroidal magnetic field ( $\mathrm{B}=0.8,1.25$ and $2.5 \mathrm{~T}$ ). The central factor governing the physics of the density limit in stellarators was demonstrated to be the decreasing net power to the plasma when the centrally peaked radiated power density profile exceeds that of the deposited power density. The process was further accelerated by the peaking of electron density under these conditions. In discharges with $\mathrm{B}=2.5 \mathrm{~T}$, simulations of the centrally peaked radiation power density profiles could be shown to be due to peaked
\end{abstract}


impurity density profiles. Laser blow off measurements clearly inferred an inward pinch of the injected aluminium. These discharges had the electron density profile form found in the improved confinement $\mathrm{H}$ NBI mode on W7-AS.

The aim of producing steady state discharges at the highest possible density in stellarators is naturally of special interest for reactor operation. Such a scenario has been best achieved in H-mode discharges, in which ELM's restricted the impurity influx to the plasma and an equilibrium in the plasma parameters with suitably low radiation power levels was possible. A density scan in ECRH discharges highlights the need to control impurity sources and choose electron densities well below the density limit in order that steady state operation can be attempted in discharges without ELM's.

A simple model of bulk radiation predicted that the limiting density should depend on the square root of heating power and this was experimentally confirmed. The magnetic field scaling of the limiting density found experimentally is attributed in this simple model to the term concerning the radial profile of the impurity density, which in turn is a function of the diffusion coefficient and inward pinch of the impurity ions. It is also expected that the dependence of the ion temperature on magnetic field must also be influential and the consequences of this dependence for neoclassical impurity ion transport requires further analysis. 


\section{Introduction}

Density limit experiments in both tokamaks and stellarators are relevant to the future operation scenario of a reactor, as sustainable steady state high density discharges will be required for power handling and ignition (JANESCHITZ et al., 1995).

In a stellarator, the maximum value of density for operation has particular importance. In fusion research the parameter of interest is the fusion triple product, $\mathrm{n}_{e} \tau_{E} \mathrm{~T}_{i}$. The condition for ignition is given by the Lawson criterion that the produced power exceeds that needed to sustain the plasma:

$$
n_{e} \tau_{E} \gtrsim 7 \times 10^{19} \mathrm{~m}^{-3} \mathrm{keVs}^{-1}
$$

where $\mathrm{n}_{e}$ is the electron density and $\tau_{E}$ the energy confinement time. The optimum product is expected near the operational density limit. Empirical studies of the energy confinement time in stellarators have been summarized in the form of an international scaling law, ISS-95. In this scaling law, the energy confinement time is given by (STROTH et al., 1996) :

$$
\tau_{I S S 95}=0.079 a^{2.21} R^{0.65} P_{\text {in }}^{-0.59} \bar{n}_{e}^{0.51} B_{o}^{0.83} t_{2 / 3}^{0.4}
$$

with units in s, m, MW, $10^{19} \mathrm{~m}^{-3}$ and T. From this scaling law the following expression is deduced :

$$
n_{e} \tau_{E} \propto a^{2.21} R^{0.65} P_{i n}^{-0.59} \bar{n}_{e}^{1.51} B_{o}^{0.83} t_{2 / 3}^{0.4}
$$

Expressing the operational density limit, $\mathrm{n}_{c}$, as :

$$
n_{e} \leq n_{c}
$$

then the maximum product obtained at the density limit can be described by :

$$
\left(n_{e} \tau_{E}\right)_{\max } \propto \bar{n}_{c}^{1.51}
$$

for fixed device parameters and heating power. Thus the identification and understanding of the density limit is a critical issue in stellarator research because it is ultimately closely related to reactor performance. In addition, a stellarator plasma is free from current disruption even in the final phase of the radiation collapse. Owing to this characteristic feature, details of the processes in radiation collapse could be observed in stellarators. Therefore, with 
this advantage the precise physics mechanism of the density limit phenomena could be determined.

A comparison of the physics of density limit operation in stellarators and tokamaks highlights the necessity of a good understanding of the various elements that contribute towards successful steady state operation. The aim of this paper is to summarize the physics aspects of density limit discharges in a stellarator and show that these results have consequences for steady state operation.

Results concerning density limit operation in tokamaks are conventionally presented in the form of an operational diagram (SUTTRop et al., 1997) or a Hugill plot (GREENWALD et al., 1988). In a Hugill plot for low beta, circular machines with a large aspect ratio the value of Murakami number $\bar{n}_{e} \mathrm{R} / \mathrm{B}$ is plotted versus $5 / \pi 1 / \mathrm{q}$, where $\bar{n}_{e}$ is the line averaged density, $\mathrm{R}$ the major radius, $\mathrm{B}$ the toroidal magnetic field and $\mathrm{q}$ the safety factor. Density limit experiments on W7-AS are summarized with data from other machines in Fig. 1 where the ASDEX data are taken from (STÄBLER et al., 1992), the ASDEX Upgrade data are taken from (MERTENS et al., 1996) and the Heliotron-E data are taken from (SuDo et al., 1990). Discharges with a Murakami parameter greater than the Greenwald limit were achieved transiently in W7-AS in this set of discharges. Similarly plasma parameters yielding values above the Greenwald limit in tokamak density limit discharges have also been achieved transiently using pellet injection (MERTENS et al., 1996) or for a duration of $0.8 \mathrm{~s}$ with only a slow increase of radiated power (DE VRIES et al., 1998).

The physics of density limits in tokamaks involves such elements as energy detachment, an edge thermal instability leading to a MARFE, particle detachment and disruption at the density limit below a critical edge temperature and above a critical edge density (MERTENs et al., 1997). The detachment and MARFE sometimes precedes the disruption and sometimes not. The density limit is also often cited as the reason for the termination of H-mode discharges in tokamaks, where the plasma makes a transition back into the $\mathrm{L}$ mode before disruption. Although a variety of phenomena takes place, the simple empirical form (Hugill diagram or Greenwald limit) is accepted to be valid in tokamak density limit events with few exceptions.

In this article a survey is made of the phenomenology of the density limit in the W7-AS stellarator. In W7-AS, the density limit is characterized by a strong degradation of diamagnetic energy and the increment of the radiation power in time even in discharges where the line integrated density is held 
constant. Particle and energy transport also play a role as the radiation collapse is approached. Experiments were performed with ECRH heating by $140 \mathrm{GHz}$ gyrotrons, NBI heating or both simultaneously.

\section{Survey of density limit phenomena}

W7-AS is a low-shear, modular stellarator with 5 periods, a major radius of $2 \mathrm{~m}$ and an effective minor radius smaller than $0.18 \mathrm{~m}$. Experiments were performed with ECRH heating by $140 \mathrm{GHz}$ gyrotrons (up to $1 \mathrm{MW}$ ), NBI heating (up to $2 \mathrm{MW}$ ) or both simultaneously. The magnetic field was varied in the range of 0.8 to $2.5 \mathrm{~T}$. The rotational transform is for most discharges is in the vicinity of 0.34 , where the configuration is characterized by 10 poloidal limiters. In the H-mode discharge, the rotational transform is in the vicinity of 0.56 , where the configuration is characterized by a separatrix.

The following types of experiments are discussed in the following subsections and the main results are briefly summarized :

(i) In NBI discharges, magnetic fields of $0.8 \mathrm{~T}, 1.25 \mathrm{~T}$ and $2.5 \mathrm{~T}$ and power inputs of 0.5 MW and 1.0 MW were chosen. Different density limit mechanisms of the plasma were observed. An oscillation in radiated power and diamagnetic stored energy is reported and its similarity to the "breathing" in Heliotron/torsatron devices is discussed. A scaling law of the maximum line averaged density reached with input power and magnetic field is derived and compared to that deduced for Heliotron-E.

(ii) In the density plateau experiments with NBI heating the impact of discharge duration on the achievable maximum density is stressed.

(iii) Combined ECRH and NBI experiments aimed at sustaining discharges at densities well below the highest possible density are presented and these experiments also show an increase of radiated power over the length of the discharge. The achievable density limit is also found to be a function of the discharge duration.

(iv) In addition, experiments with a ramp down in ECRH power are detailed and the evolution of a collapse on a slow enough time scale for measurements to be carried out are presented. The shrinking of the 
minor plasma radius with decreasing net power could be measured. In these experiments an experimental cooling curve from radiating impurity ions is obtained and a comparison of the temperature dependence of the radiated power loss with transport losses is made.

(v) It can be shown that the key to understanding the oscillating density limit discharges lies with the sudden ejection of plasma density.

(vi) The cooling curve of impurity ions in a variety of density limit discharges is obtained experimentally. In the comparison of ECRH and NBI discharges, a surprising degree of coincidence is found in the experimental cooling curve of discharges with 0.5 MW heating.

(vii) In H-mode experiments with a density scan for discharges with 0.5 MW and 1.0 MW ECRH power, it is shown that a H-mode with ELM's is a possible means of achieving steady state operation.

The diagnostics used to monitor the plasma parameters included a YAG laser system capable of yielding electron temperature and density profiles at $50 \mathrm{~ms}$ intervals and a 24 channel ECE radiometer for electron temperature profiles with a time resolution of $1 \mathrm{~ms}$. Electric field measurements in the edge plasma were inferred from the electron impact excited spectral triplet Boron IV lines ( $2 \mathrm{~s} 3 \mathrm{~S}, 2 \mathrm{p} 3 \mathrm{P}$ odd at $2821 \AA$ ). All 3 lines of the triplet are observed simultaneously on 5 spatial channels with a maximum time resolution of $300 \mathrm{~Hz}$. The observation geometry is close to the elliptical plane, so that only the poloidal rotation component is measured. Doppler line broadening and line shift are fitted to evaluate chord integrated ion temperatures and poloidal rotation velocities. Convolution by the instrumental function and finite slit widths is calculated analytically and taken into account. An Abel inversion of these line integrated measurements yields radial profiles of ion temperatures and rotation velocities. For the determination of the radial electric field, the ion pressure gradient term and a higher order term stemming from the finite lifetime of the excited B IV electronic state are calculated and taken into account. The total radiated power is deduced from Abel inverted measurements of the line integrated power flux onto $4 \mu \mathrm{m}$ gold foil absorbers of a 32 channel bolometer array.

The radial profiles of the diffusion coefficient, D, and inward pinch velocity, $v$, of impurities were calculated from measurements of the time evolution 
of soft X-ray emission and $\mathrm{Al}$ impurity lines after aluminium impurity injection by laser blow-off (BURHENn et al., 1997). The soft X-ray measurements are carried out with 2 surface barrier diode cameras, each of 36 channels. The placement of a $12.5 \mu \mathrm{m}$ beryllium foil in front of the entrance slit allows the filtering out of energy components below $1 \mathrm{keV}$. The $\mathrm{Al}$ impurity lines were also measured by a Jobin-Yvon spectrometer ( Al XI ) and a Bragg spectrometer ( Al XII and Al XIII ). The soft X-ray emission with and without impurity injection are subtracted to yield the net emission due to impurity injection.

\subsection{Magnetic field and power scan in NBI plasmas}

A magnetic field and power scan were performed in NBI plasmas. The magnetic field, B, was set at the values of $0.8 \mathrm{~T}, 1.25 \mathrm{~T}$ and $2.5 \mathrm{~T}$ and at each of these magnetic fields the NBI heating of $0.5 \mathrm{MW}$ and $1 \mathrm{MW}$ was applied. The time evolution of the diamagnetic energy, line integrated density, central electron temperature and total radiated power for these discharges is shown in Fig. 2.

These discharges share the common feature that initially the diamagnetic energy decreases with time. This is even observed in discharges where $\bar{n}_{e}$ can be held at a constant value. These density plateau experiments with NBI heating are discussed in detail in the next section. Even though cooling of the plasma core and an increase in the radiated power from impurity ions is expected, the observed increase in radiated power during the discharge has also been shown to be due to centrally peaked impurity density profiles with equilibrium times in excess of the discharge length (GIANnONE et al., 1999).

In these discharges at a particular value of $\bar{n}_{e}$, it can be seen that a dramatic decrease in the diamagnetic energy and $\bar{n}_{e}$ occurs which may or may not lead to termination of the discharge. Increasing $\bar{n}_{e}$ leads to a more rapid decrease in diamagnetic energy, so that the discharge length before collapse is influenced by the choice of $\bar{n}_{e}$. Extending the operation of W7-AS plasmas with NBI heating alone at a power greater than $0.5 \mathrm{MW}$ presents the further difficulty that beam fuelling of the plasma leads quickly to rising $\bar{n}_{e}$ and reaching of the density limit for operation.

In discharges at $\mathrm{B}=1.25 \mathrm{~T}$, the dramatic decrease of diamagnetic energy and recovery was most pronounced. At first a decrease in diamagnetic energy and increase in radiated power were observed. At the maximum of radiated power there was a sharp decrease in $\bar{n}_{e}$. Shown in Fig. 3 is the 
time evolution of the radial electric field measurements at various radii. The rapid decrease of the radiated power is therefore connected both with the sudden ejection of plasma density and rapid change of radial electric field from strongly negative values to values in the vicinity of zero. At the density limit, the radiation instability collapse continues and the temperature becomes lower than a critical value, where symmetry-breaking perturbations of temperature and potential on the magnetic surface become unstable. The growth of these perturbations leads to rapid rapid plasma loss takes place (ITOH et al., 2000).

These experiments share many common features with the first long pulse experiments on LHD (TAKEIRI et al., 1999). The feature of plasma radius contraction inferred from visible emission observations of tangential views on LHD can be confirmed by results from ECRH power ramp down experiments in W7-AS discussed in a later section. Detailed power balance calculations would be needed to test the hypothesis whether or not such cyclical behavior can be explained by the time dependent NBI power absorption and radiated power leading to a varying location of the plasma boundary with consequences for neutral penetration depth, density profile evolution and impurity ion flux.

Steady state operation of stellarators is a final goal. The first experiments of $20 \mathrm{~s}$ duration on LHD with $0.5 \mathrm{MW}$ NBI heating at $1.5 \mathrm{~T}$ for $\bar{n}_{e}$ above $5 \times 10^{19} \mathrm{~m}^{-3}$ show oscillations with a period of 1 to 3 seconds in the plasma temperature, plasma density and impurity radiation (TAKEIRI et al., 1999). With increasing line integrated density during an oscillation it was noted that the density profile peaks. At densities of $3 \times 10^{19} \mathrm{~m}^{-3}$ such phenomena were not observed. A comparison of the phenomena observed in this discharge and similar discharges in W7-AS shows that the observations on LHD and W7-AS have many common features.

The maximum achievable density in density limit experiments from the magnetic field and power input variation over the last four years of operation are summarized in Fig. 4 and these measurements extend earlier results (Giannone et al., 1997; Grigull et al., 1998). The critical density, $\mathrm{n}_{c}$, is then fitted by a power law fit with respect to the volume averaged absorbed power, $\mathrm{P}_{a b s} / \mathrm{V}_{p}\left[\mathrm{MW} / \mathrm{m}^{3}\right]$, and $\mathrm{B}[\mathrm{T}]$ :

$$
n_{c}=1.46 \pm 0.07\left(P_{a b s} / V_{p}\right)^{0.48 \pm 0.03} B^{0.54 \pm 0.05}\left[10^{20} m^{-3}\right]
$$

The limiting line averaged density for the planned W7-X stellarator assuming 
$\mathrm{R}=5.5 \mathrm{~m}, \mathrm{a}=0.55 \mathrm{~m}, \mathrm{P}_{a b s}=20 \mathrm{MW}$ and $\mathrm{B}=2.5 \mathrm{~T}$ is predicted to be $1.4 \times 10^{20} \mathrm{~m}^{-3}$ and the planned HSR stellarator reactor with $\mathrm{P}_{a b s}=600 \mathrm{MW}$, $\mathrm{B}=5.0 \mathrm{~T}$ and $\mathrm{V}_{p}=1400 \mathrm{~m}^{3}$ is predicted to be $2.3 \times 10^{20} \mathrm{~m}^{-3}$. The estimate for HSR is consistent with the requirements of the projected ignition scenario (BEIDLER et al., 1998). This form of scaling law is very similar to that found in Heliotron-E density limit experiments, where $\mathrm{n}_{c} \propto\left(\mathrm{P}_{a b s} / \mathrm{V}_{p}\right)^{0.5} \mathrm{~B}^{0.5}$ was reported (Sudo et al., 1990).

Theoretically, assuming a simplified radiation model and one dimensional transport to describe the energy balance, it is possible to predict the scaling of $\mathrm{n}_{c}$ with respect to the volume averaged absorbed power in stellarators (ITOH and ITOH, 1988) :

$$
\bar{n}_{e}^{2}<K P_{a b s} / V_{p}
$$

where the coefficient $\mathrm{K}$ is given as :

$$
K^{-1}=2 \int_{0}^{1} L(Z, T)\left(\frac{n_{Z}}{n_{e}}\right)\left(\frac{n_{e}}{\bar{n}_{e}}\right)^{2} x d x
$$

where $\mathrm{L}(\mathrm{Z}, \mathrm{T})$ is the steady state cooling rate (POST et al., 1977). In this model the plasma is assumed to be core radiation dominated, and the coefficient $\mathrm{L}(\mathrm{Z}, \mathrm{T})$ is assumed to increase very strongly as the temperature becomes lower than some critical temperature. In this simplified situation, the density limit scales with $\mathrm{P}^{0.5}$. In contrast, it was predicted that the density limit by detachment based on radiation losses at the plasma edge is linearly proportional to power. The magnetic field scaling of the limiting density found experimentally would naturally be influenced by changes in the value of $\mathrm{K}$ resulting from the change in $\mathrm{B}$. Firstly, the term has a factor concerning the radial profile of the impurity density, which in turn is a function of the diffusion coefficient and inward pinch of the impurity ions. Also, it has been shown that the diffusion coefficient is itself a function of density and heating power (BURHENN et al., 1998). In experiments with $\mathrm{P}_{E C R H}=0.48 \mathrm{MW}$ and central densities of $3.5 \times 10^{19} \mathrm{~m}^{-3}$ and $7.0 \times 10^{19} \mathrm{~m}^{-3}$ at $\mathrm{B}=2.5 \mathrm{~T}$ the diffusion coefficient was reduced at all radii by a factor of 3 by increasing the density. Secondly, the term has a factor concerning the peaking of the density profile. It is found from YAG measurements that the density profile form is identical so that it is expected that the term concerning impurity profile peaking dominates. Moreover, it can be shown that inclusion of the thermal conductivity in the power balance equations and assumptions about the B 
dependence of the thermal conductivity can lead to density limit scaling laws with an explicit magnetic field dependence (ITOH et al., 2000; SuEnder and WoBIG, 1998). This simplified model then seems to capture some essential element of physics features of the density limit on W7-AS.

\subsection{Density plateau sustained by NBI}

The survey in the previous section clearly demonstrates the presence of various time scales in the dynamical evolution of density limit discharges. Next, long time scale discharges up to $2 \mathrm{~s}$ are considered. It is shown in Fig. 5 that the maximum operating density seems to depend on the duration of the discharge. At higher densities the discharges are closer to the critical temperature for collapse. The impurity density profile requires a longer period at a lower density to reach the concentration required to cause the radiation power levels needed for collapse (GiAnNONE et al., 1999). This illustrates another issue in the steady state operation of stellarators near the density limit.

In W7-AS, a density plateau discharge with constant line integrated electron density reaches a maximum in diamagnetic energy, then with rising total radiated power the diamagnetic energy falls until the discharge either terminates or recovers to start the cycle once again. The line integrated electron density defines therefore the length of the discharge and so the density limit in W7-AS decreases as the required discharge length is increased. At sufficiently low densities, discharges of duration up to $2 \mathrm{~s}$ were possible. In discharges at successively higher density plateau values, the rate of diamagnetic energy decrease after the peak in diamagnetic energy was larger at higher densities. Decreasing net power to the plasma due to core radiation is shown to be responsible for the observed fall in diamagnetic energy. The plasma cooling and increasing radiated power is reinforced by the observed peaking of the electron density profiles since the net heating power per particle is even further decreased. These density plateau discharges are closely related to the improved confinement scenario in W7-AS (H-NBI mode) but at densities in the vicinity of the density limit (STroth et al., 1998). The increase in radiated power during density plateau discharges was found to be caused by a steady increase in centrally peaked profiles of the radiation power density. Such a peaking in the radiation profiles has been shown to be due to a peaking of the impurity ions (GIANNONE et al., 1999) and has also been observed previously in the W-VIIA stellarator (W-VIIA TEAM and 
NI Group, 1985) and Heliotron-E (KANEKo et al., 1987). In the WVII-A stellarator the observations could be mainly accounted for by neoclassical impurity transport of oxygen ions from the vessel walls and partially by the deposition of carbon, oxygen and tantalum ions deposited centrally by the NI beams. In W7-AS NBI discharges at $2.5 \mathrm{~T}, 3 \%$ shine through losses and $2 \%$ charge exchange losses were calculated. Impurity ions, produced by energetic ions impinging on the wall, could therefore have been a relevant factor in the observed discharge evolution. In addition, in W7-A it has been calculated that sputtering due to charge exchange losses could be an equally important source of impurity ions.

The neoclassical theory used to simulate the results in the W-VIIA experiments is a one dimensional code solving the basic set of coupled continuity equations for the ion impurity density. It assumes axisymmetry for the classical, Pfirsch-Schlüter and plateau fluxes corresponding to collisions of the impurity ions with the background plasma. The non-axisymmetric contributions can be shown to be negligible for the case of small helical ripple and large aspect ratio. One aspects of the simulation requires further development. The inclusion of radial electric field effects on classical transport fluxes is still needs to be implemented for a truly universal neoclassical simulation of impurity transport in a stellarator.

In neoclassical theory the radial flux of impurity ions, $\Gamma_{Z}$, with charge $\mathrm{Z}$ is given by the sum of diffusive and convective components :

$$
\Gamma_{Z}=-D_{\perp}^{Z} \frac{d n_{Z}}{d r}+v_{\perp}^{Z} n_{Z}
$$

where $D_{\perp}^{Z}$ and $v_{\perp}^{Z}$ are the diffusivity and convection velocity respectively. The density and temperature gradient of the background ions determines the convection velocity :

$$
v_{\perp}^{Z}=D_{\text {neo }}\left(\eta \frac{d n_{i}}{d r}+\theta \frac{d T_{i}}{d r}\right)
$$

with $\mathrm{D}_{\text {neo }}, \eta$ and $\theta$ being a function of $\mathrm{n}_{i}, \mathrm{~T}_{i}$, the safety factor and the toroidal magnetic field.

Using neoclassical transport theory for ions and impurities in ohmically heated discharges in tokamaks (TOKAR et al., 1997), an accumulative instability for high Z impurities has been identified. It is driven by the neoclassical pinch term with the gradient in ion density leading to an inward pinch and 
the gradient in ion temperature in tokamaks to an outward pinch. The latter term is called temperature screening as it reduces the tendency for the peaking of impurities. This is shown to be unstable as a reduction in ion temperature and consequently its gradient, leads to a reduction of the outward driven term and thus a peaking of the ion density. This in turn drives a further increase in the ion density gradient and the inwards driven flux by this gradient. An ion temperature drop from the increase in ion density therefore reinforces the initial perturbation. Increased diffusive particle flux from the peaked ion density and reduced conductive heat losses from the plasma core due to temperature profile flattening are stabilizing factors. Neoclassical convection and radiation losses are the destabilizing factors. A quantitative criterion is given for a critical central density above which this mechanism is predicted to be unstable. Such a corresponding mechanism for stellarators, should not be of concern as the ion temperature gradient driven component of the convection velocity is also directed inwards.

In addition to the peaking of the impurity density profile, the change of the electron density profile could also be important in the process of the peaking of the radiation profile. On W7-AS it has been observed that the density profile reacts to the electron temperature profile (STROTH et al., 1999). With on-axis and off-axis ECRH heating the electron density profile was flattened and peaked respectively in response to the different temperature profiles. For on-axis heating when the temperature profile is increasingly peaked, this can be understood in terms of enhanced temperature gradient driven particle fluxes leading to flatter density profiles. For off-axis heating, in the absence of temperature gradients for not so peaked profiles then with a reduction of temperature gradient driven particle flux the density profiles can remain peaked. In the density limit discharges, when the central electron temperature gradient decreases due to the strong radiation loss, then the net inward pinch of electrons would be expected to become larger, causing a more peaked density profile and more peaked central radiation. This link could also enhance the thermal instability. The presence of temperature gradient driven particle fluxes in NBI plasmas must be experimentally confirmed in future experiments.

\subsection{Combined ECRH and NBI heating}

For steady state operation it is therefore clear that in the vicinity of the density limit difficulties arise from impurity radiation at electron tempera- 
tures where the radiated power per impurity ion rises rapidly. If the radiation loss is the key for understanding density limit phenomena, then the observed operational density limit might depend on the heating method as NBI and ECRH discharges are characterized by different temperature and density profiles. The origin of a dependence of radiation loss on the heating method could possibly arise from differences in the edge plasma parameters leading to different impurity fluxes.

It was therefore instructive to consider discharges significantly below the density limit with central temperatures well above those measured near the density limit. Such a discharge is shown in Fig. 6. With a similar central electron density of $6 \times 10^{19} \mathrm{~m}^{-3}$, distinct differences in the discharges were observed. In NBI heated discharges (red) significantly more radiated power than in the ECRH discharges (blue) was measured. Occasionally spikes in the emission from Fe XVI lines were observed and it is conjectured that fast ion loses from the beam are responsible for the almost doubling in magnitude of radiated power in comparison to the ECRH discharge. Another possible cause for this significant difference in radiated power and hence impurity concentration in the plasma is that NBI heated plasmas may be additionally injecting trace amounts of light impurities (W-VIIA TEAM and NI Group, 1985). However in light of different densities and temperatures in the discharges, that moreover are not in a steady state, a more systematic investigation is necessary.

Even at these reduced densities, it can be seen in Fig. 7 that the radial profiles of radiated power are centrally peaked and slowly increasing. In the NBI discharges these profiles are more distinctly peaked than the ECRH discharges. The central electron temperature is higher and more peaked and the density profile broader in the ECRH discharge.

With combined ECRH and NBI heating, the rise of $\bar{n}_{e}$ seen towards the end of the NBI discharge can be controlled. Even though the total radiated power rises relatively slowly in comparison to the previously discussed NBI or ECRH discharges, the radial profiles of radiated power continue to rise slowly but surely in the vicinity of the plasma minor axis. Even in this case, it can be seen from the non-equilibrium status of the radiated power measured by the soft X-ray silicon barrier diodes that the time constant for the impurity density to reach an equilibrium is much longer than the duration of the discharge (BURHENn et al., 1998). For steady state operation such a scenario is possibly interesting since the radiated power fraction is only approximately $20 \%$. 


\subsection{Radiation collapse in ECRH plasmas with power ramp down}

Details of the radiation collapse in the density limit phenomena was investigated on W7-AS by performing power ramp down experiments in ECRH plasmas. This study takes advantage of the feature of current disruption free operation in stellarators. Even during a radiation driven collapse, the confining magnetic surfaces are not destroyed.

From an initial ECRH power of 0.5 MW, the deposited power was ramped down to $50 \mathrm{~kW}$ over a period of $0.7 \mathrm{~s}$. With a central electron density of $6 \times 10^{19} \mathrm{~m}^{-3}$, well below the cut-off density for the $140 \mathrm{GHz}$ gyrotrons, the deposited power is centrally deposited for the chosen magnetic field of $2.5 \mathrm{~T}$ with a typical FWHM of $3 \mathrm{~cm}$. In Fig. 8 an overview of the plasma parameters are shown. As expected, a decrease in diamagnetic energy with time due to decreasing net power input to the plasma is observed. As the plasma core cools, a stage is eventually reached where the radiated power rapidly increases.

Although the plasma at low heating power is only optically thick for ECE above about $300 \mathrm{eV}$ for the central densities of $7 \times 10^{19} \mathrm{~m}^{-3}$, it is nevertheless interesting to view the time evolution of the electron temperature profiles measured by ECE with steps of 10 milliseconds. As shown in Fig. 9, towards the end of the discharge distinctly hollow electron temperature profiles can be inferred from the ECE measurements. Furthermore, with decreasing net power to the plasma, the plasma radius clearly contracts. In contrast to tokamaks, where a contracting plasma radius can lead to a major disruption if, for example, the $\mathrm{q}=2$ magnetic surface in the plasma becomes MHD unstable, this plasma contraction does not lead to any sudden dramatic termination of the plasma. Indeed Fig. 2 shows the possibility that the plasma can recover if the plasma can be sustained long enough so that impurities are ejected, thereby allowing the net power to once again reach a value comparable to the deposited power.

These experiments are evaluated to provide information about the power balance in the radiation collapse. Fig. 10 illustrates the various channels of power flow as a function of stored energy, W. The power balance equation is written as :

$$
\frac{d W}{d t}=P_{a b s}-P_{t r a n s}-P_{r a d}
$$


where $\mathrm{P}_{\text {rad }}$ is the radiated power loss and $\mathrm{P}_{\text {trans }}$ is the remaining power loss, considered mainly to be due to transport processes. The deposited power, $\mathrm{P}_{a b s}$, in NBI discharges is calculated from a Monte Carlo code simulating the NBI heating on W7-AS and in ECRH discharges it is approximated by the ECRH power. The above equation is rewritten to obtain the relation for $\mathrm{P}_{\text {trans }}$ and $\mathrm{P}_{\text {in }}$ that is plotted in Fig. 10 :

$$
P_{\text {trans }}=P_{a b s}-\frac{d W}{d t}-P_{\text {rad }}=P_{\text {in }}-P_{\text {rad }}
$$

where $\mathrm{W}$ is given by the measured diamagnetic signal, $W_{\text {dia }}$. This plot clearly shows that the two loss channels, $\mathrm{P}_{\text {trans }}$ and $\mathrm{P}_{\text {rad }}$, have an opposite dependence on $\mathrm{W}$ (or temperature) for conditions with constant density), and that there is a very sharp peak in the radiation loss in the low temperature limit. The negative slope of $\mathrm{P}_{\text {rad }}$ with respect to $\mathrm{W}$ (or temperature) is fitted from the experimental data as :

$$
P_{\text {rad }} \propto\langle T\rangle^{-1}
$$

in the final stage of collapse, with $\mathrm{P}_{\text {rad }}>50 \mathrm{~kW}$ and the derived mean plasma temperature for this ECRH plasma is estimated by :

$$
\left\langle T_{e}(e V)\right\rangle=2 W_{d i a} /\left(3 e \bar{n}_{e} V_{p}\right)
$$

where $V_{p}$ is the plasma volume and :

$$
W_{d i a}=\frac{3}{2} 4 \pi^{2} R \int_{0}^{a}\left(n_{e} T_{e}+n_{i} T_{i}\right) r d r
$$

is the measured diamagnetic energy. In writing equation $14, \mathrm{~T}_{e} \gg \mathrm{T}_{i}$ is assumed because an ECRH plasma at low density is under consideration. However, when studying the NBI heated plasmas where electron-ion coupling at higher densities means that $\mathrm{T}_{e} \approx \mathrm{T}_{i}$, then the derived temperature must be divided by an additional factor of 2 . The negative slope of the radiated power versus temperature is the origin of the radiation collapse as a decrease in temperature is reinforced by increased radiation and further cooling. It is also noted that $\mathrm{P}_{\text {trans }}$, also fitted from the experimental data, shows the following dependence on the derived mean temperature :

$$
P_{\text {trans }} \propto\left(W_{\text {dia }} / \bar{n}_{e}\right)^{2.3} \propto\langle T\rangle^{2.3}
$$

In addition, when power balance is dominated by $\mathrm{P}_{\text {trans }}$ then : 


$$
P_{\text {trans }}=n T V_{p} / \tau_{E}
$$

so that combining the two equations above yields :

$$
\tau_{E} \propto P^{-\frac{\alpha-1}{\alpha}}
$$

with $\alpha=2.3$. This relation could be interpreted as $\tau_{E} \propto \mathrm{P}^{-0.57}$ in the limit where transport loss is dominant. This dependence is consistent with the ISS95 scaling law.

\subsection{Oscillation near density limit}

By evaluating the experimental cooling curve, insight into the mechanisms of the oscillation near the density limit can be obtained. In order to sustain the oscillatory evolution in the stored energy for fixed NBI power, there should be some nonlinear (hysteresis) relation between the loss terms and diamagnetic energy. It is shown that such a hysteresis exists predominantly in the relation $\mathrm{P}_{\text {rad }}(\mathrm{W})$ and not in $\mathrm{P}_{\text {trans }}(\mathrm{W})$.

The total radiated power versus $\left\langle\mathrm{T}_{e}\right\rangle$ is shown in Fig. 11 and the cyclical path of the discharges that recover from a plasma contraction resulting from decreasing net power to the plasma is underlined. The trajectory is remarkably reproducible for the $0.5 \mathrm{MW}$ NBI discharge with $\mathrm{B}=1.25 \mathrm{~T}$.

$\mathrm{P}_{\text {trans }}(\mathrm{W})$ is calculated from the power balance equation of equation 12 with $\mathrm{P}_{a b s}=\eta \mathrm{P}_{N B I}$ and $\eta$ is the heating efficiency of injected NBI power. In Fig. 12, assuming $\eta=0.76$ both $\mathrm{P}_{\text {rad }}(\mathrm{W})$ and $\mathrm{P}_{\text {trans }}(\mathrm{W})$ are plotted and the hysteresis nature of $\mathrm{P}_{\text {rad }}(\mathrm{W})$ is clearly illustrated. In contrast, within errors $\mathrm{P}_{\text {trans }}(\mathrm{W})$ is a single valued function during the cycle of diamagnetic energy. It is concluded that the limit cycle oscillation of the stored energy near the density limit is due to the hysteresis in $\mathrm{P}_{\text {rad }}(\mathrm{W})$.

The steady state cooling rates, $\mathrm{L}(\mathrm{Z}, \mathrm{T})$, for low density, high temperature plasmas in a corona model with the absence of transport effects have been tabulated (POST et al., 1977) :

$$
P_{Z}=n_{e} n_{Z} L(Z, T)
$$

where $\mathrm{P}_{Z}$ is the radiative loss rate from impurity ions of charge $\mathrm{Z}$ per unit volume, $\mathrm{n}_{e}$ is the electron density and $\mathrm{n}_{Z}$ is the impurity ion density. This equation can be rewritten in the form : 


$$
\frac{P_{Z}}{n_{e}^{2}}=\frac{n_{Z}}{n_{e}} L(Z, T)
$$

The right hand side contains two important components. One is the relative impurity concentration and the other is the cooling rate of an impurity ion which is a function of electron temperature and impurity species. Experimentally, dividing the total radiated power measured by the bolometers and dividing this by $\mathrm{V}_{p}$ and the square of $\bar{n}_{e}$ then one arrives at the normalized radiated power :

$$
\frac{P_{r a d}}{V_{p}\left(\bar{n}_{e}\right)^{2}}=\frac{n_{Z}}{n_{e}} L(Z, T)
$$

From equation 19 it can be seen that $\mathrm{P}_{\text {rad }}(\mathrm{W})$ is dependent on the electron density, $\mathrm{n}_{e}$, the relative impurity concentration, $\mathrm{n}_{Z} / \mathrm{n}_{e}$ and $\mathrm{L}(\mathrm{Z}, \mathrm{T})$. It is noted that in arriving at Equation 21, variables on the right hand side with dependencies on the local values of density and temperature are replaced with volume average quantities on the left hand side. Even though the local radiated power is a sum over impurities and the concentrations in the various ionization states, it is nevertheless possible to realize interesting comparisons of the impurity content of discharges by plotting the normalized radiated power versus the mean derived temperature. In addition it is possible to use such a plot to highlight the mechanism responsible for the hysteresis in $\mathrm{P}_{\text {rad }}(\mathrm{W})$.

In Fig. 13, the normalized radiated power and $\mathrm{P}_{\text {rad }}$ for the collapsing discharge at $\mathrm{B}=1.25 \mathrm{~T}$ and $\mathrm{P}_{N B I}=0.5 \mathrm{MW}$ are plotted as a function of the derived mean plasma temperature for NBI plasmas, assuming $\mathrm{T}_{e} \approx \mathrm{T}_{i}$, and in this case :

$$
\left\langle T_{e}(e V)\right\rangle=W_{d i a} /\left(3 e \bar{n}_{e} V_{p}\right)
$$

It can be seen that the hysteresis in normalized radiated power is much smaller than in $\mathrm{P}_{\text {rad }}$. Therefore, there is only a small difference in normalized radiated power in the phases where the plasma is collapsing $(\mathrm{dW} / \mathrm{dt}<0)$ or where the plasma is recovering $(\mathrm{dW} / \mathrm{dt}>0)$. The hysteresis in $\mathrm{P}_{\text {rad }}(\mathrm{W})$ is attributed to the difference in electron density in the collapsing and recovering phase. The physics of the mechanism that causes the oscillation of radiated power is a rapid ejection of density when the plasma is cooled down. As the normalized radiated power shows little hysteresis in the collapsing or 
recovering phase it can be concluded that the impurity concentration rise during a cycle is not the basis for the observed hysteresis in $\mathrm{P}_{\text {rad }}(\mathrm{W})$. A theoretical treatment of the critical conditions for onset of the ejection of density and subsequent oscillation is presented in a separate publication (ITOH et al., 2000).

\subsection{Comparison of ECRH and NBI plasmas}

The normalized radiated powers for ECRH and NBI plasmas are now compared. Shown in Fig. 14 are the results of this comparison for the ECRH power ramp discharge and the NBI discharges at $1.25 \mathrm{~T}$ and $2.5 \mathrm{~T}$ shown in Fig. 2. At these relatively low electron densities the time scale for the increase of impurity density in the plasma centre with time is much longer than the ramp down time of the ECRH power. In Fig. 14, it can be seen that the value of $n_{Z} / \mathrm{n}_{e}$ in the ECRH discharge and the NBI discharges overlap and show the same rise in normalized radiated power with decreasing mean derived temperature. It must be concluded that there is no substantial difference of the relative impurity concentration or species in the ECRH and NBI plasmas for the discharges with 0.5 MW heating. In Fig. 6, the differences in radiated power seen are therefore simply due to the differences in the radial profiles and evolution of the temperature of each discharge. A comparison of these experimental cooling curves and a theoretical one for carbon with a relative carbon concentration of 0.05 is shown in Fig. 14(a). Although a volume averaged quantity is compared to one dependent on the local values of density and temperature, it is suggested that such a plot is valuable for comparing the impurity content of different discharges because a comparison is possible even when the individual impurity species concentrations are not available.

\subsection{H-mode in NBI and ECRH plasmas}

Discharges with 0.5 MW NBI heating ( $0.38 \mathrm{MW}$ deposited ) at $2.5 \mathrm{~T}$ and at an edge iota value, $\iota(\mathrm{a})$, of 0.553 and 0.557 are compared in Fig. 15. The transition into a H-mode in W7-AS has been shown to be a sensitive function of $\iota(\mathrm{a})$ and can be explained in terms of the absence of low order resonances in the edge region inside the separatrix leading to minimum in magnetic pumping and hence to reduced damping of plasma poloidal rotation (WAgner et al., 1994; Wobig and Kisslinger, 1997). In Fig. 15 this 
small difference in $\iota$ (a) leads to the two very different behaviours in total radiated power as the discharge with $\iota(\mathrm{a})=0.557$ makes a transition to the $\mathrm{H}$-mode at the time indicated by the dotted line while the discharge with $\iota(\mathrm{a})=0.553$ does not. The rapid rise of both the total radiated power and the power measured by the 36 channel soft X-ray camera with $12.5 \mu \mathrm{m}$ Be filter reflects the improvement in confinement of impurity ions in addition to the improvement for deuterium ions. Effectively, a density limit in the H-mode discharge is reached as the radiated power increases to values greater than the nominal input power. The comparison of these two discharges dramatically illustrates the fact that the maximum in diamagnetic energy is attained at two different densities because the impurity concentration in both discharges is much higher in the H-mode.

The path to steady state confinement in W7-AS therefore cannot be found in ELM free H-mode discharges. Shown in Fig. 16 is a line integrated density scan in a 1.0 MW ECRH plasma at $2.5 \mathrm{~T}$ and $\iota(\mathrm{a})=0.564$. There exists a density threshold for the H-mode transition in W7-AS. Below this density threshold a discharge with H-mode features will not be observed (GRIGULL et al., 1999). Therefore only the discharges at the two highest densities in the 1.0 MW ECRH discharges are in the H-mode. As indicated by the $\mathrm{H}_{\alpha}$ traces these discharges have considerably less ELM activity than those discharges at the lower density.

At the higher ECRH power for discharges without a transition to the $\mathrm{H}$-mode, it is evident that the soft X-ray radiated power does not reach equilibrium. The discharges at the two lower densities are optimized confinement ECRH discharges (Stroth et al., 1998; BALDZUHN et al., 2000) and the radiated power increases strongly during the discharge. At the lower input power the radiated power does not increase strongly because the discharge is no longer in the improved confinement mode. However, for those discharges in the H-mode with significant ELM activity the radiated soft X-ray power does reach equilibrium. The radial profiles of radiated power from the 32 channel bolometer array rises continuously with a peaked profile in the discharges at the lower densities while at the higher densities the peaked profiles appear to have almost reached a steady state.

The conjecture that the increase in the radiated power of the discharges in the improved optimum confinement mode may be due to an increase in impurity flux during the discharge is not substantiated by the line integral bolometer measurements. These measurements show a constant level of radiated power in the plasma edge for the duration of the discharge. The radiated 
power increases only in the channels viewing the plasma centre. Simulations with the transport code ASTRA (PEREVERZEV et al., 1991) and impurity transport code STRAHL (BEHRINGER, 1987) show that a ramp in the impurity flux at the plasma edge is characterized by a discernible increase in the radiated power at the plasma edge.

It is concluded that the path to steady state confinement in W7-AS will be, like tokamaks (ASDEX TEAM, 1989; HorTON et al., 1999), through such H-mode discharges with strong ELM activity. This assumes that such a scenario is compatible with machine operation in the presence of power flux bursts on the plasma facing components.

The addition of a divertor to W7-AS is planned and should lead to a reduction in the impurity fluxes to the plasma. Density limit experiments in W7-AS with a divertor then should achieve sustainable discharges at higher values of density as the onset of plasma cooling may not lead to radiated power levels that are comparable to the input power.

\section{Conclusions}

A magnetic field and power scan of NBI heated plasmas for density limit discharges in the W7-AS stellarator was carried out. The radiation collapse of the plasma was in each case provoked by centrally peaked radiation profiles increasing in time. This result can be understood in terms of the measured diffusion and inward pinch coefficient for impurity ion transport. Radial electric field measurements showed that the ejection of plasma density and impurities occurs simultaneously with the sudden change of the radial electric field from strongly negative values to values in the vicinity of zero.

A comparison of discharges with NBI, ECRH or combined heating was carried out. It could be demonstrated that for these discharges, each with approximately the same line integrated density, apparent differences in total radiated power can be understood in terms of the difference in temperature evolution of each discharge. Combined heating realized improved density control.

The condition of detachment in stellarators has been analyzed theoretically (ITOH and ITOH, 1988). Scaling studies confirm the square root dependence on power as predicted for the bulk radiation dominated density limit discharges in W7-AS. The magnetic field scaling of the limiting density which is found experimentally might be a consequence of the dependence of 
the transport coefficients of impurities or plasma energy on magnetic field. Further theoretical analysis is required to reach a conclusive explanation.

From ECRH power ramp down experiments it could be inferred that the density limit in stellarators is arrived at through power balance of input power and radiated power. When the radiated power approaches the total input power, the plasma undergoes disruption free contraction. The plasma cooling and increasing bulk radiation is further accelerated by the peaking of the electron density. In the case that the impurity ion concentration is reduced by bulk plasma density ejection, the discharge is able to recover. The cyclic behavior of diamagnetic energy versus net input power is an interesting feature that highlights the interdependence of the density limit, radiated power and impurity transport.

The observation that an ELM free H-mode initiates a radiation collapse and that discharges in a H-mode with strong ELM activity reach an equilibrium state with respect to the radial profiles of radiated power within the $2 \mathrm{~s}$ duration of the discharge, suggests that the latter scenario, like tokamaks, will be the path to steady state confinement in W7-AS.

In this study on W7-AS, the role of radiation loss in the density limit phenomena is clarified. The experimental cooling curve is obtained and a coexistence of the radiation loss and transport loss, which have opposite temperature dependences, is observed. Two types of density limit phenomena are identified. A simple density limit, which is characterized by the monotonous increase of radiation loss in time, and a self-sustained oscillation of radiation loss. In the latter oscillation events, the hysteresis curve of $\mathrm{P}_{\text {rad }}(\mathrm{W})$ is observed, and such a feature stems from the rapid ejection of electrons in the cooled plasma rather than the selective loss or accumulation of impurities. The difference between a monotonous collapse and radiation oscillation seems to be precisely the respective absence or presence of sudden electron density loss in the cooled plasma. The boundary of the onset of ejection of electrons has not yet been identified, and research into the details must be continued. These experiments share many common features with the first long pulse experiments on LHD (TAKEIRI et al., 1999). The feature of plasma radius contraction inferred from visible emission observations of tangential views on LHD can be confirmed by results from ECRH power ramp down experiments in W7-AS. Detailed power balance calculations would be needed to test the hypothesis whether or not such cyclical behavior can be modelled by the time dependent NBI power absorption and radiated power. The resulting varying location of the plasma boundary would have consequences for neutral 
penetration depth, density profile evolution and impurity ion flux.

\section{Acknowledgements}

Two of the authors (KI and SII) acknowledge the hospitality of the Alexander von Humboldt Stiftung $(\mathrm{AvH})$ and Max-Planck-Institut fur Plasmaphysik during their stay in Germany. The technical expertise of H. Wolf in the design and commissioning of the bolometer array was an invaluable part of this research. 


\section{References}

ASDEX TeAm (1989). Nucl. Fusion 29, 1959-2040.

Baldzuhn J., Giannone L., Kick M., et al. (2000). Plasma Phys. Controlled Fusion ??, ???

Behringer K. (1987). Description of the Impurity Transport Code STRAHL. Report JET-R(87)08, JET.

Beidler C., Stroth U., and Wobig H. (1998). Empirical Scaling Laws and Extrapolation to Helias Reactors. Technical report, IPP 2/338.

Burhenn R., Anton M., Baldzuhn J., et al. (1998). Impurity transport investigations at W7-AS Stellarator. In Journal of Plasma Fusion Research SERIES, 8th International Toki Conference on Plasma Physics and Controlled Nuclear Fusion, Toki, Japan, page 255.

Burhenn R. et al. (1997). 24th European Conference on Controlled Fusion and Plasma Physics (Berchtesgaden) IV, 1659.

de Vries P., Rapp J., Schuller J., and Tokar M. (1998). Phys. Rev. Lett. 80, 3519.

Giannone L., Bellido E., Brakel R., et al. (1997). 24th European Conference on Controlled Fusion and Plasma Physics (Berchtesgaden) IV, 1565 .

GiAnnone L. et al. (1999). Journal of Nucl. Materials 266-269, 501.

Greenwald M., Terry J., Wolfe S., et al. (1988). Nucl. Fusion 28, 2199 .

Grigull P., Giannone L., Stroth U., et al. (1998). Density Limit Study on the W7-AS Stellarator. In Journal of Plasma Fusion Research SERIES, 8th International Toki Conference on Plasma Physics and Controlled Nuclear Fusion, Toki, Japan, page 291.

Grigull P., Hirsch M., MCCormick K., et al. (1999). 26th European Conference on Controlled Fusion and Plasma Physics (Maastricht) 23J, 1473. 
Horton L., Sartori R., et al. (1999). Nucl. Fusion 39, 993.

Itoh K., Itoh S., and Giannone L. (2000). Plasma Phys. Controlled Fusion ??, ???

Iтон K. and Ітон S.-I. (1988). J. Phys. Soc. Jpn. 57, 1269.

Janeschitz G. et al. (1995). Plasma Phys. Controlled Fusion 37, 11A, A19.

Kaneko H., Kondo K., Motojima O., et al. (1987). Nucl. Fusion 37, 1075.

Mertens V. et al. (1996). 23rd European Conference on Controlled Fusion and Plasma Physics (Kiev) I, 15.

Mertens V. et al. (1997). Nucl. Fusion 37, 1607.

Pereverzev G., Yushmanov P., Dnestrovskit A., Polvoi A., TarasJAN K., and Zakharov L. (1991). An Automatic System for Transport Analysis in a tokamak. Report IPP 5/42, Max Planck Institut für Plasmaphysik.

Post D., Jensen R., Tarter C., Grasberger W., and Lokke W. (1977). Atomic Data and Nuclear Tables 20, 397.

Stäbler A., McCormick K., Mertens V., et al. (1992). Nucl. Fusion $32,1557$.

Stroth U. et al. (1996). Nucl. Fusion 36, 1063.

Stroth U. et al. (1998). Plasma Phys. Controlled Fusion 40, 779.

Stroth U., Geist T., Koponen J. P. T., Hartfuss H. J., and Zeiler P. (1999). Phys. Rev. Lett. 82, 928.

Sudo S., TAkeiri Y., Zushi H., et al. (1990). Nucl. Fusion 30, 11.

Suender D. and Wobig H. (1998). Bifurcation of Temperature in 3-D Plasma Equilibria. Technical report, IPP 2/342.

Suttrop W. et al. (1997). Plasma Phys. Controlled Fusion 39, 2051. 
Takeiri Y., Noda N., Kaneko O., et al. (1999). 26th European Conference on Controlled Fusion and Plasma Physics (Maastricht) 23J, 1365.

Tokar M., Rapp J., Bertschinger G., et al. (1997). Nucl. Fusion 27, 1698.

W-VIIA Team and NI Group (1985). Nucl. Fusion 25, 1593.

Wagner F. et al. (1994). Plasma Phys. Controlled Fusion 36, A61.

Wobig H. and Kisslinger J. (1997). 24th European Conference on Controlled Fusion and Plasma Physics (Berchtesgaden) II, 315. 


\section{List of Figures}

1 Greenwald limit diagram for density limit discharges in ASDEX and ASDEX Upgrade tokamaks and Heliotron-E and W7-AS stellarators. . . . . . . . . . . . . .

2 Overview of density limit discharges with a) $\mathrm{P}_{N B I}=0.5 \mathrm{MW}$ and b) $\mathrm{P}_{N B I}=1.0 \mathrm{MW}$ at a rotational transform, $\iota$, of 0.34 . A collapse in the diamagnetic energy coincides with a rise in radiated power. . . . . . . . . . . . . . 30

3 Time evolution of radial electric field measurements at various radii showing that the rise in radiated power and the concurrent plasma cooling is accompanied by a reduction in the magnitude of the radial electric field. . . . . . . . . . .

4 Power law fit of $\bar{n}_{e}$ with volume averaged absorbed power and magnetic field for density limit discharges in W7-AS. The fit formula is given by $\mathrm{n}_{c}=1.46 \pm 0.07\left(\mathrm{P}_{a b s} / \mathrm{V}_{p}\right)^{0.48 \pm 0.03}$

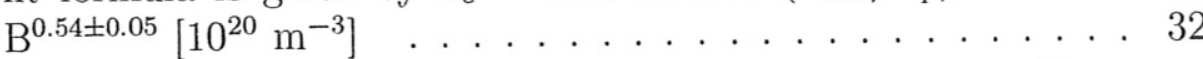

5 Evolution of 0.5 MW NBI discharges at various constant line integrated densities showing the steadily decreasing diamagnetic energy and increasing radiated power. The rate of increase in the peaked impurity density profile is a function of the transport coefficients, $\mathrm{D}$ and $\mathrm{v}_{\text {in }}$. At higher densities the discharges are closer to the critical temperature for collapse.

6 Overview of discharge evolution for a central electron density of $6 \times 10^{19} \mathrm{~m}^{-3}$ with $0.5 \mathrm{MW} \mathrm{NBI}, 0.5 \mathrm{MW}$ ECRH and combined heating of 0.5 MW NBI and 0.5 MW ECRH. Density control is possible with combined heating in comparison to the NBI discahrge.

Comparison of radial profiles of electron density, temperature and radiated power in discharges with a central electron density of $6 \times 10^{19} \mathrm{~m}^{-3}$ with $0.5 \mathrm{MW}$ ECRH (top row), $0.5 \mathrm{MW}$ NBI (middle row) and combined heating of $0.5 \mathrm{MW} \mathrm{NBI}$ and $0.5 \mathrm{MW}$ ECRH (bottom row). The increase in the centre of the radiated power profile in each discharge with time is a feature of high density discharges in stellarators. 
8 Overview of ECRH power ramp down discharges, initially with $\mathrm{P}_{\text {ECRH }}=0.5 \mathrm{MW}$, at a rotational transform, $\iota$, of 0.34 and $\mathrm{B}=2.5 \mathrm{~T}$. By decreasing the net power to the plasma in this way, the condition of increasing radiated power can be simulated. 36

9 Evolution of electron temperature profiles from ECE and radiation profiles in the collapsing phase of the ramp down in ECRH power experiments. The rise in radiated power and fall in electron temperature in a time interval of $60 \mathrm{~ms}$ can be documented as ECE measurements are possible below the cut off density for the $140 \mathrm{GHz}$ gyrotrons. . . . . . . . . . . 37

10 Evolution of total absorbed power, $\mathrm{P}_{a b s}$, radiated power, $\mathrm{P}_{\text {rad }}$ and the difference of these interpreted as the power carried by transport processes, $\mathrm{P}_{\text {trans }}$, plotted as (a) a function of diamagnetic energy or (b) time. At the density limit the radiated power approaches the absorbed power. . . . . . . . . . 38

11 Comparison of radiated power versus derived average temperature at magnetic fields of $1.25 \mathrm{~T}$ and $2.55 \mathrm{~T}$ with a) $\mathrm{P}_{N B I}=0.5 \mathrm{MW}$ or $\mathrm{P}_{E C R H}=0.5 \mathrm{MW}$ and b) $\mathrm{P}_{N B I}=1.0 \mathrm{MW}$ at a rotational transform, $\iota$, of 0.34 . The observed hysteresis is caused by a density collapse at the density limit. . . . . . .

12 Evolution of total absorbed power, $\mathrm{P}_{a b s}$, radiated power, $\mathrm{P}_{\text {rad }}$ and the difference of these interpreted as the power carried by transport processes, $\mathrm{P}_{\text {trans }}$, for the $\mathrm{P}_{N B I}=0.5 \mathrm{MW}$ discharges at (a) $\mathrm{B}=1.25 \mathrm{~T}$ and (b) $\mathrm{B}=2.5 \mathrm{~T}$ plotted as a function of time (left column) or diamagnetic energy (right column). The hysteresis nature of $\mathrm{P}_{\text {rad }}(\mathrm{W})$ is contrasted to $\mathrm{P}_{\text {trans }}(\mathrm{W})$ which within errors is a single valued function during the cycle of diamagnetic energy. . . . . . . . . . . . . . . 40

13 Total radiated power and the normalized radiated power of Eqn. 21 for the $\mathrm{P}_{N B I}=0.5 \mathrm{MW}$ discharge at $\mathrm{B}=1.25 \mathrm{~T}$ plotted as a function of derived mean temperature. The observed hysteresis in radiated power is therefore due to the density collapse at the density limit. 
14 Comparison of experimental cooling curves in discharges with $\mathrm{P}_{N B I}$ or $\mathrm{P}_{E C R H}=0.5 \mathrm{MW}$ at a rotational transform, $\iota$, of 0.34 . For comparison the cooling curve of carbon with $5 \%$ relative concentration is shown. The presentation of the measurements in this form allows a simple but rapid comparison of the impurity content of different discharges. . . . . . . . . .

15 Comparison of evolution of radiated power in discharges in the vicinity of a rotational transform, $\iota$, of 0.56 with $\mathrm{B}_{o}=2.5 \mathrm{~T}$ and $\mathrm{P}_{N B I}=0.5 \mathrm{MW}$. The $\mathrm{H}$-mode transition at the time given by the dotted line is indicated by the fall in the $\mathrm{H}_{\alpha}$ trace. . .

16 Overview of a density scan in ECRH discharges at $\mathrm{B}_{o}=2.5 \mathrm{~T}$ and $\iota(\mathrm{a})=0.564$ with $\mathrm{P}_{E C R H}=1.0 \mathrm{MW}$. Only the discharges at the two highest densities are in the H-mode. As indicated by the $\mathrm{H}_{\alpha}$ traces these discharges have considerably less ELM activity than those discharges at the lower density. . . . . . . . 44

17 Comparison of empirical stellarator scaling law (SUDo et al., 1990) for density limit discharges using W7-A, Heliotron-E and W7-AS discharges. . . . . . . . . . . . . . 45 


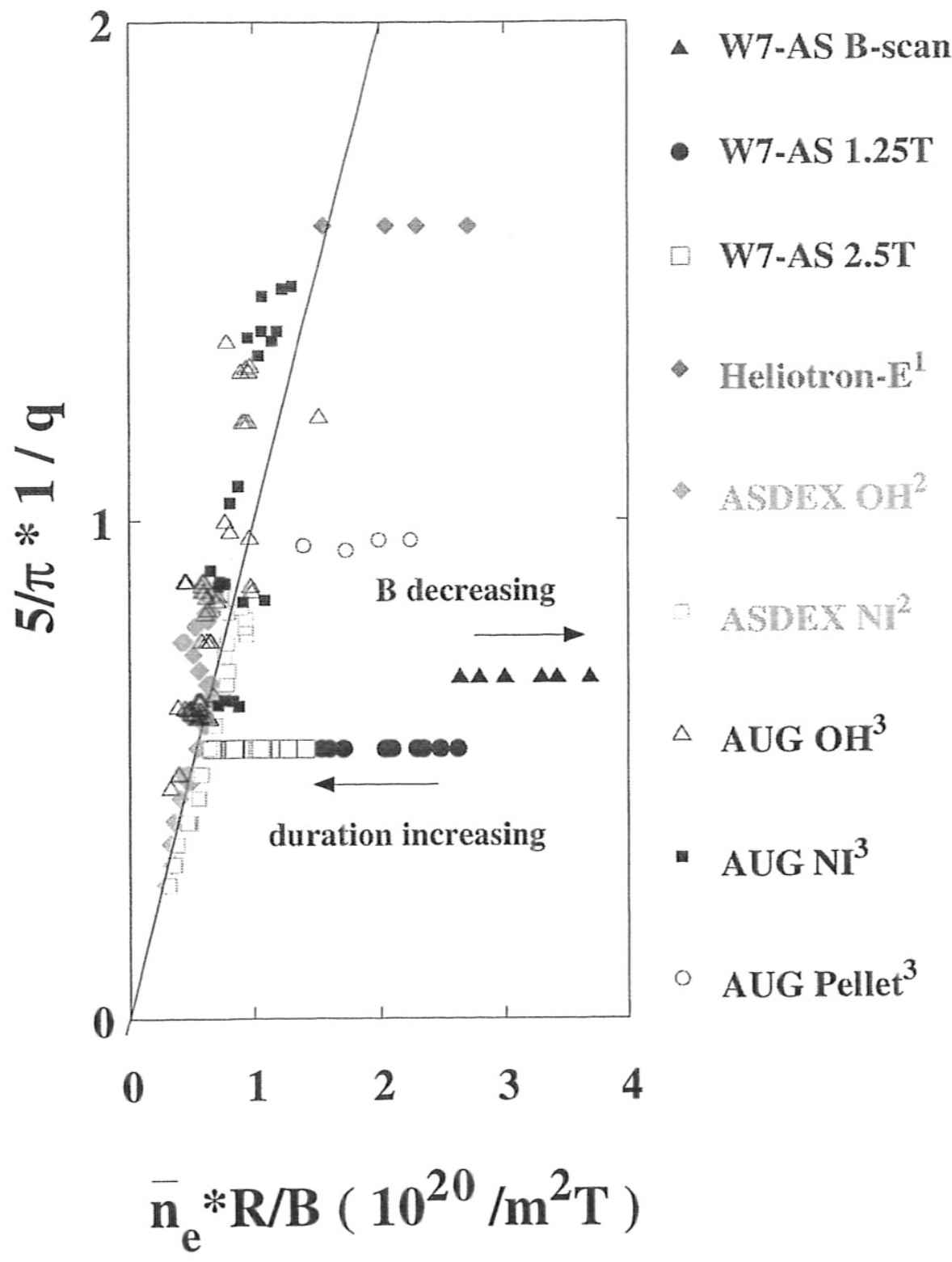

Figure 1: Greenwald limit diagram for density limit discharges in ASDEX and ASDEX Upgrade tokamaks and Heliotron-E and W7-AS stellarators. 

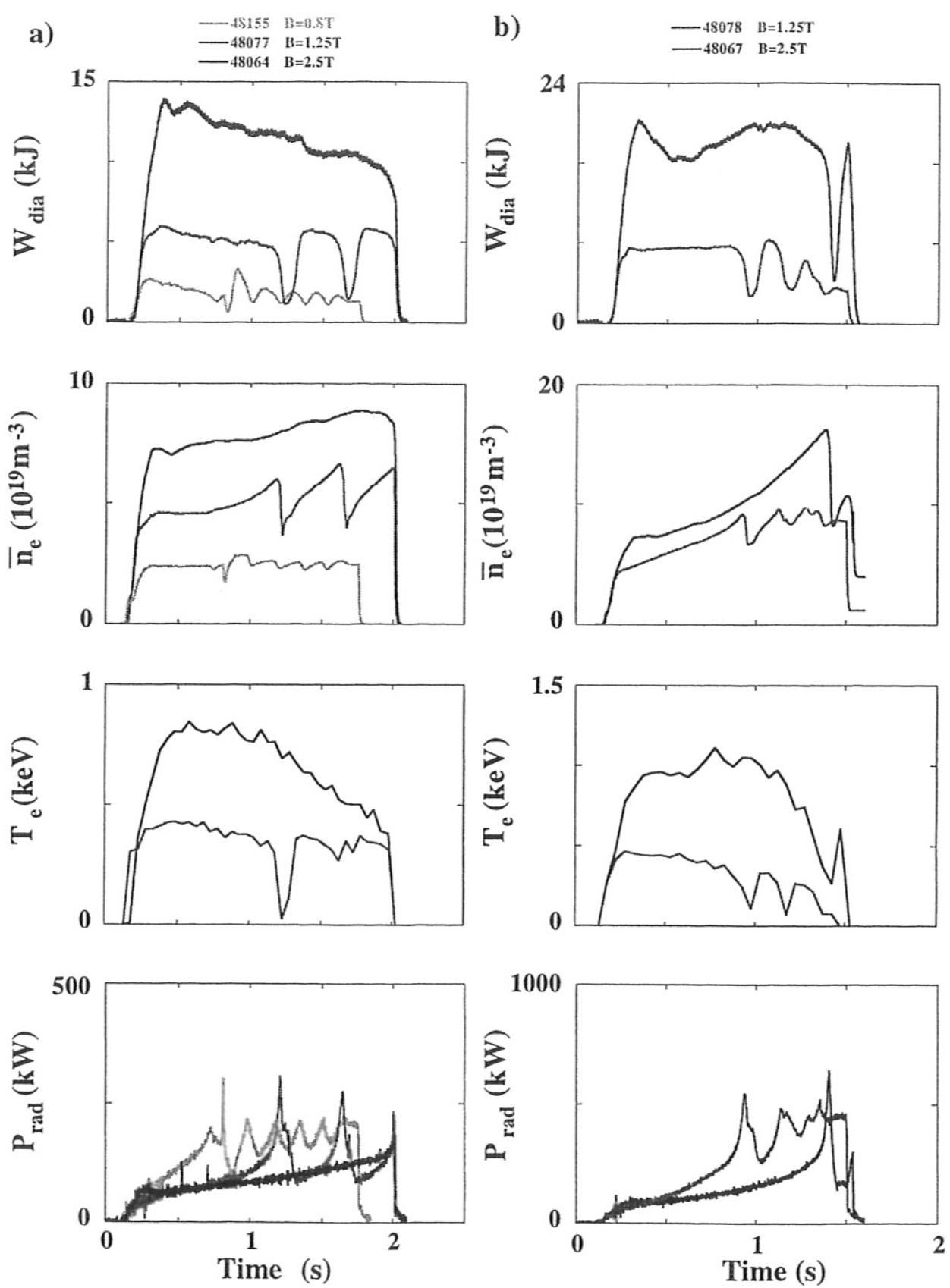

Figure 2: Overview of density limit discharges with a) $\mathrm{P}_{N B I}=0.5 \mathrm{MW}$ and b) $\mathrm{P}_{N B I}=1.0 \mathrm{MW}$ at a rotational transform, $\iota$, of 0.34 . A collapse in the diamagnetic energy coincides with a rise in radiated power. 


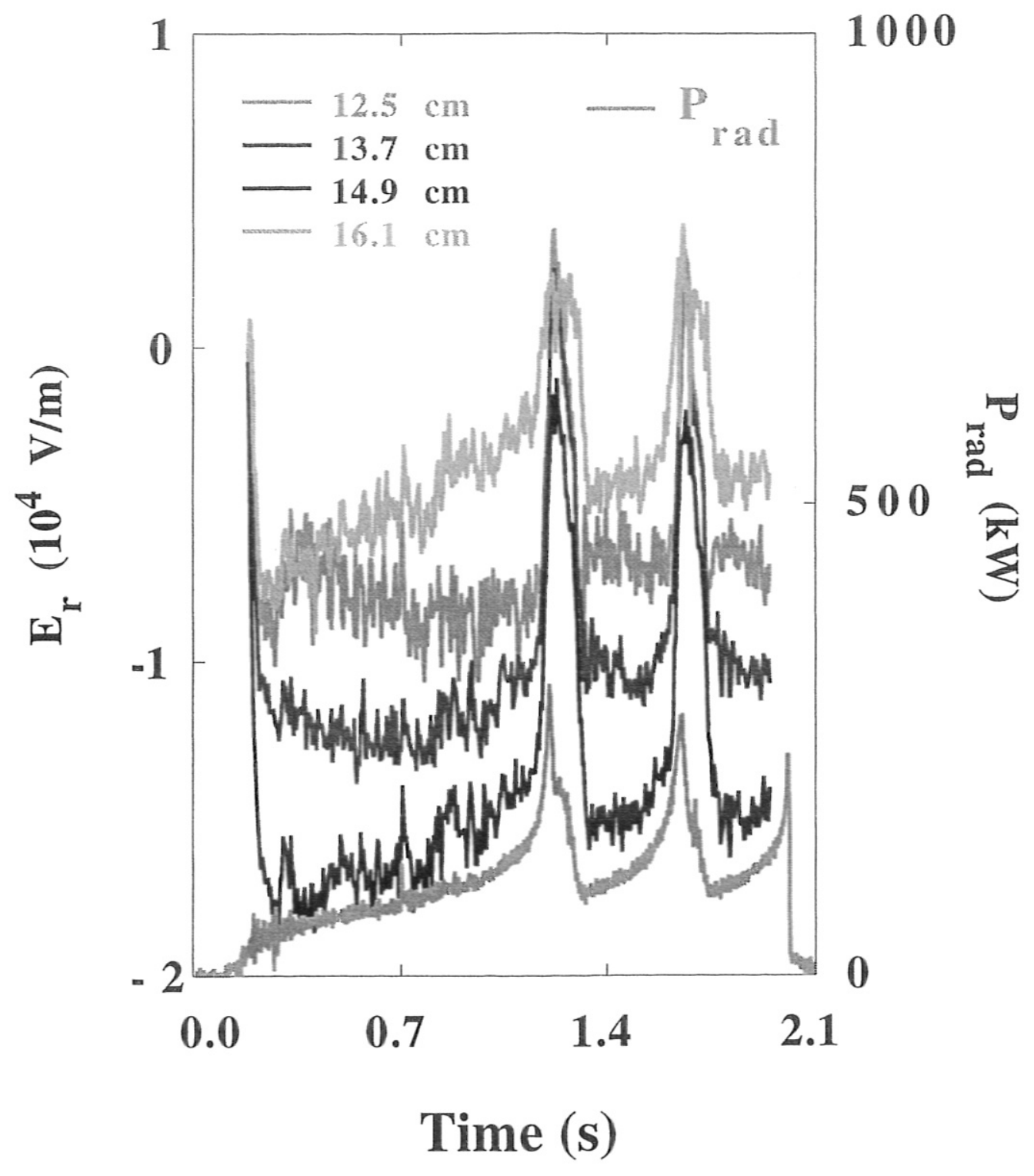

Figure 3: Time evolution of radial electric field measurements at various radii showing that the rise in radiated power and the concurrent plasma cooling is accompanied by a reduction in the magnitude of the radial electric field. 


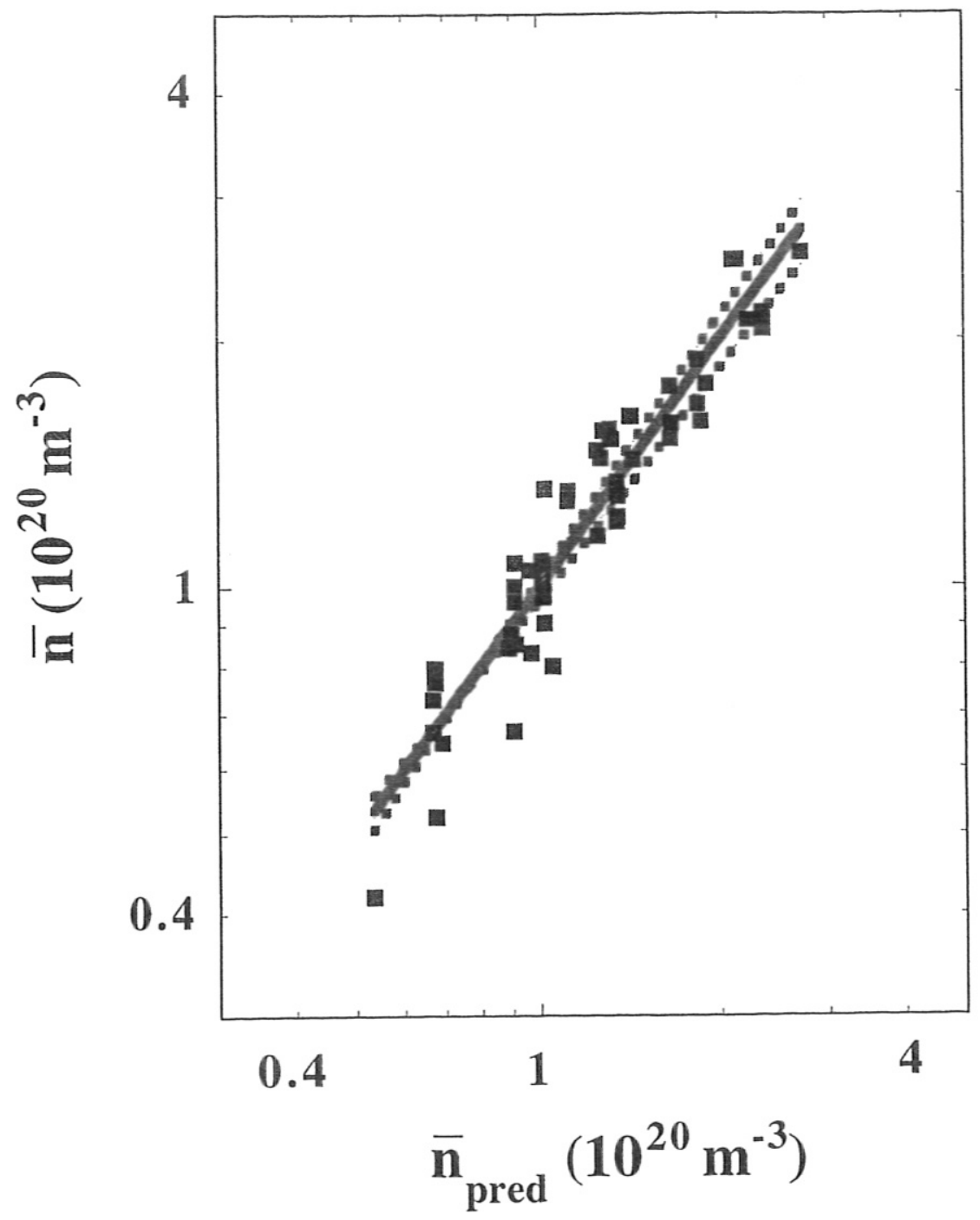

Figure 4: Power law fit of $\bar{n}_{e}$ with volume averaged absorbed power and magnetic field for density limit discharges in W7-AS. The fit formula is given by $\mathrm{n}_{c}=1.46 \pm 0.07\left(\mathrm{P}_{a b s} / \mathrm{V}_{p}\right)^{0.48 \pm 0.03} \mathrm{~B}^{0.54 \pm 0.05}\left[10^{20} \mathrm{~m}^{-3}\right]$ 

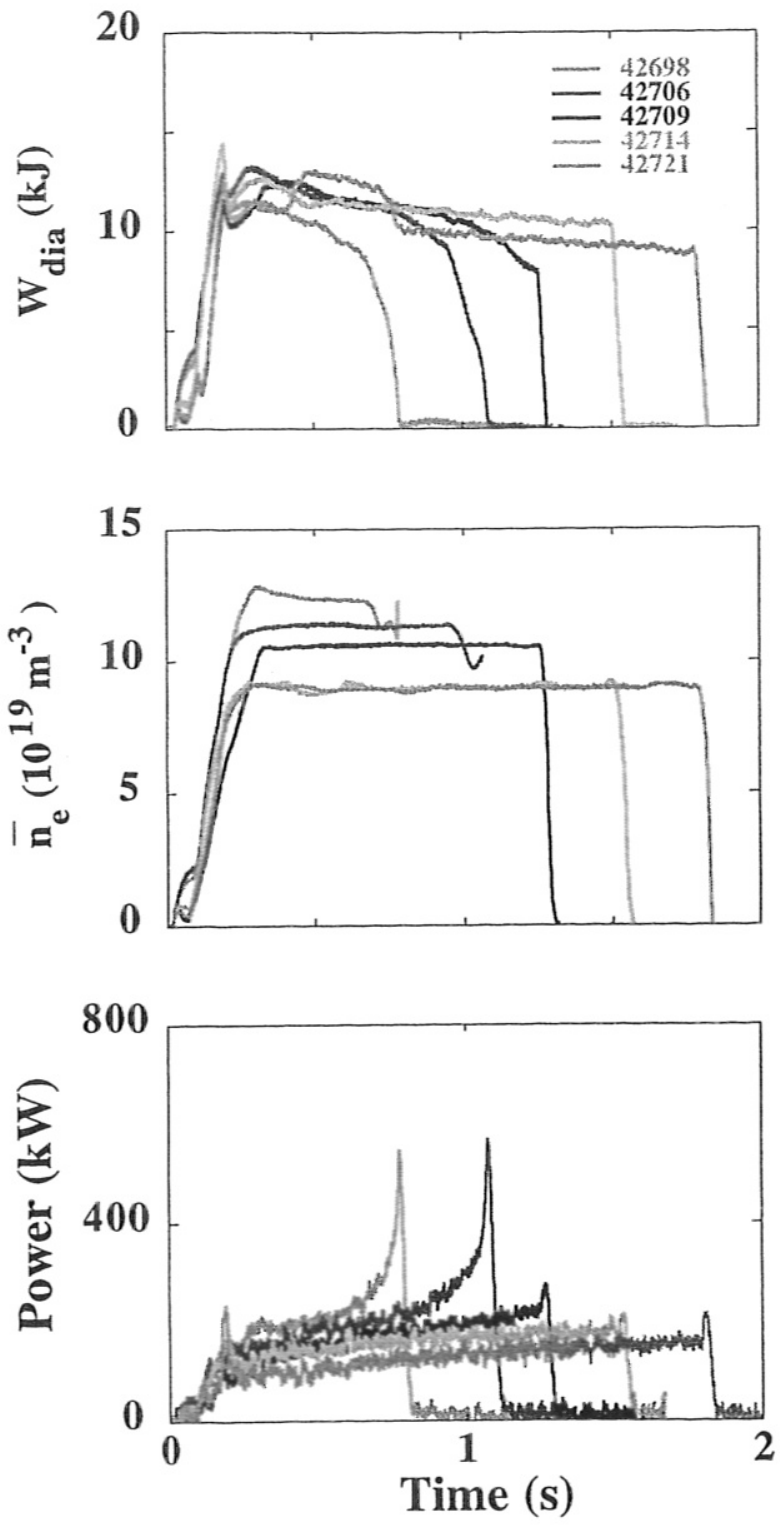

Figure 5: Evolution of 0.5 MW NBI discharges at various constant line integrated densities showing the steadily decreasing diamagnetic energy and increasing radiated power. The rate of increase in the peaked impurity density profile is a function of the transport coefficients, D and $v_{\text {in }}$. At higher densities the discharges are closer to the critical temperature for collapse. 


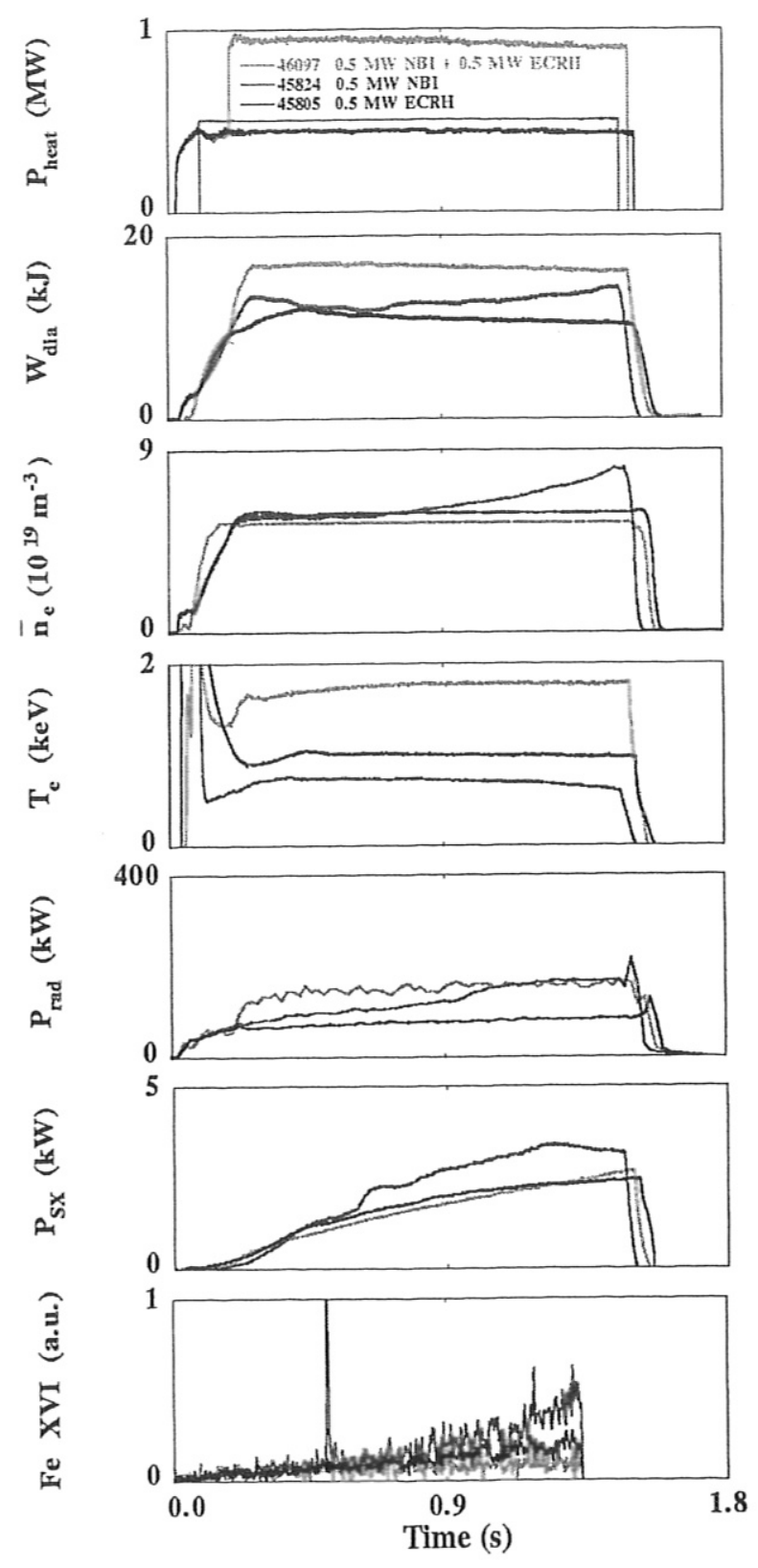

Figure 6: Overview of discharge evolution for a central electron density of $6 \times 10^{19} \mathrm{~m}^{-3}$ with 0.5 MW NBI, 0.5 MW ECRH and combined heating of $0.5 \mathrm{MW}$ NBI and 0.5 MW ECRH. Density control is possible with combined heating in comparison to the NBI discahrge. 

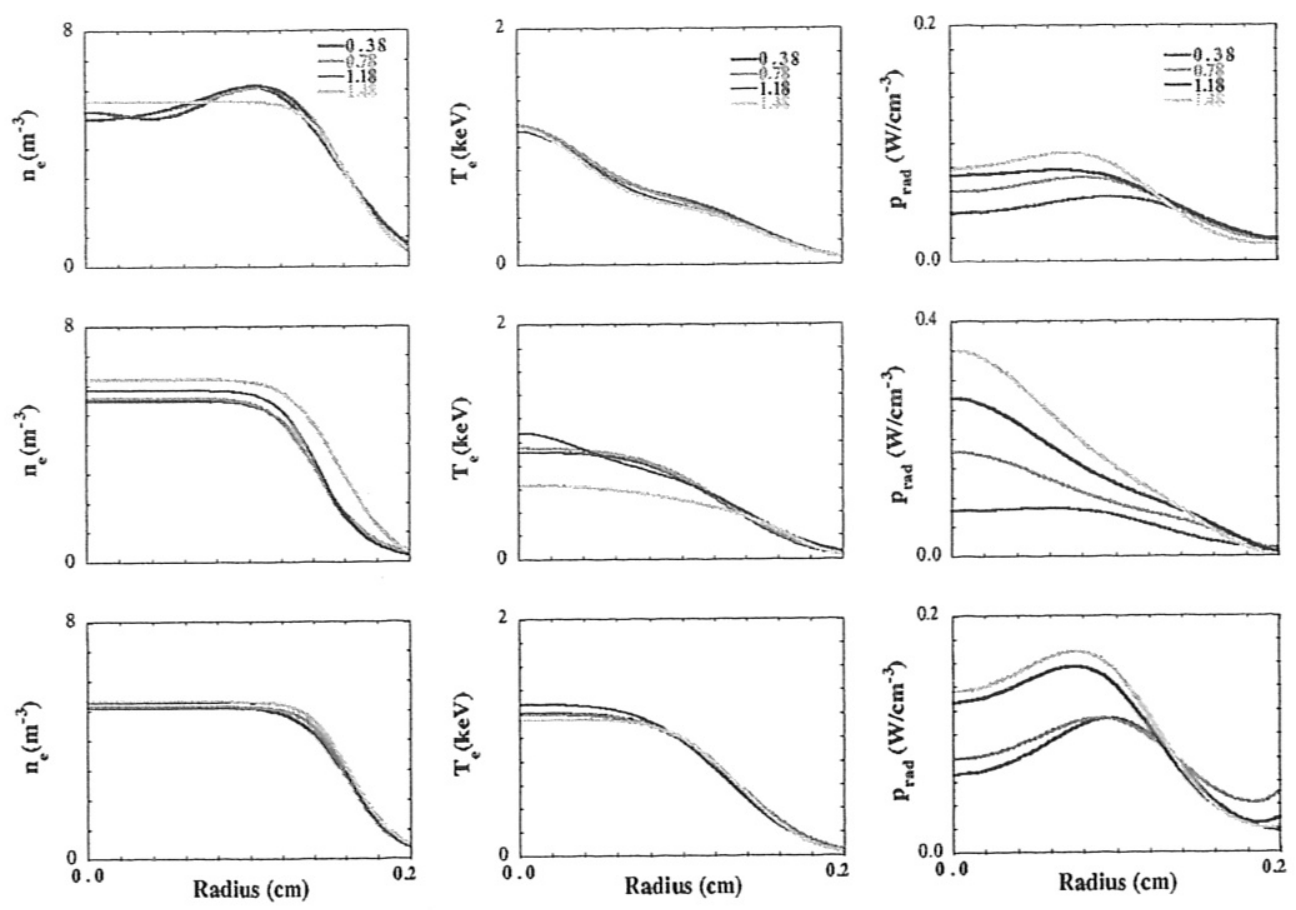

Figure 7: Comparison of radial profiles of electron density, temperature and radiated power in discharges with a central electron density of $6 \times 10^{19} \mathrm{~m}^{-3}$ with 0.5 MW ECRH (top row), 0.5 MW NBI (middle row) and combined heating of $0.5 \mathrm{MW}$ NBI and 0.5 MW ECRH (bottom row). The increase in the centre of the radiated power profile in each discharge with time is a feature of high density discharges in stellarators. 

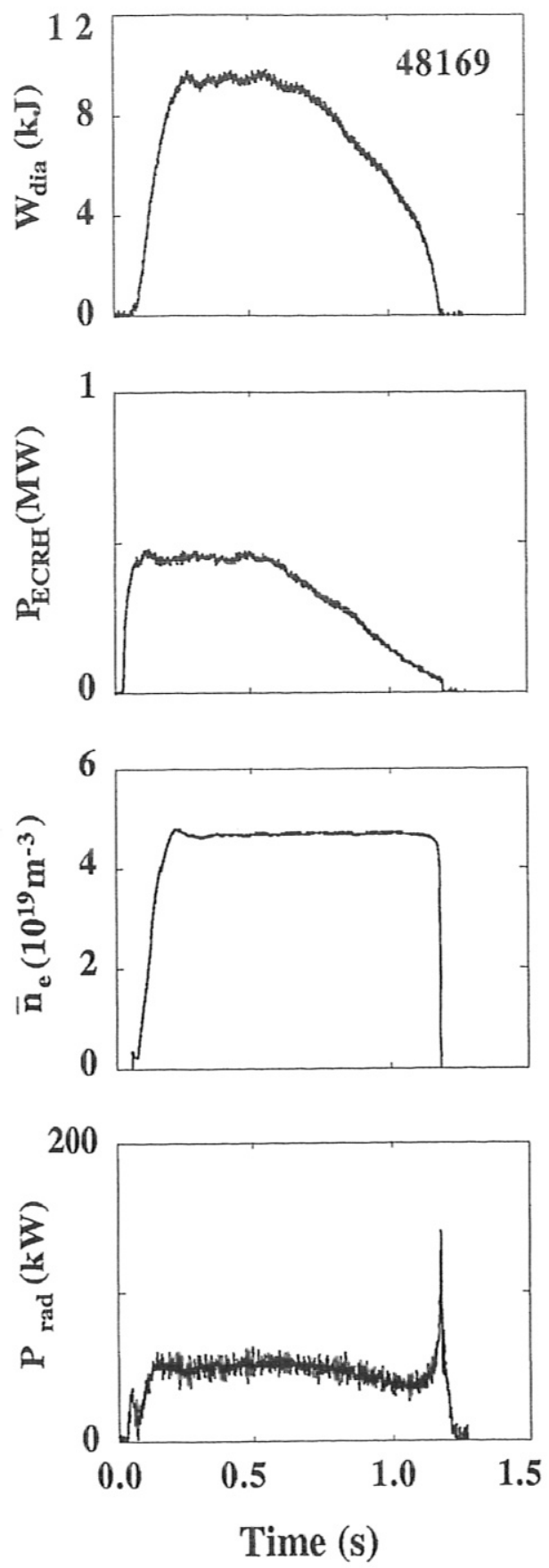

Figure 8: Overview of ECRH power ramp down discharges, initially with $\mathrm{P}_{E C R H}=0.5 \mathrm{MW}$, at a rotational transform, $\iota$, of 0.34 and $\mathrm{B}=2.5 \mathrm{~T}$. By decreasing the net power to the plasma in this way, the condition of increasing radiated power can be simulated. 

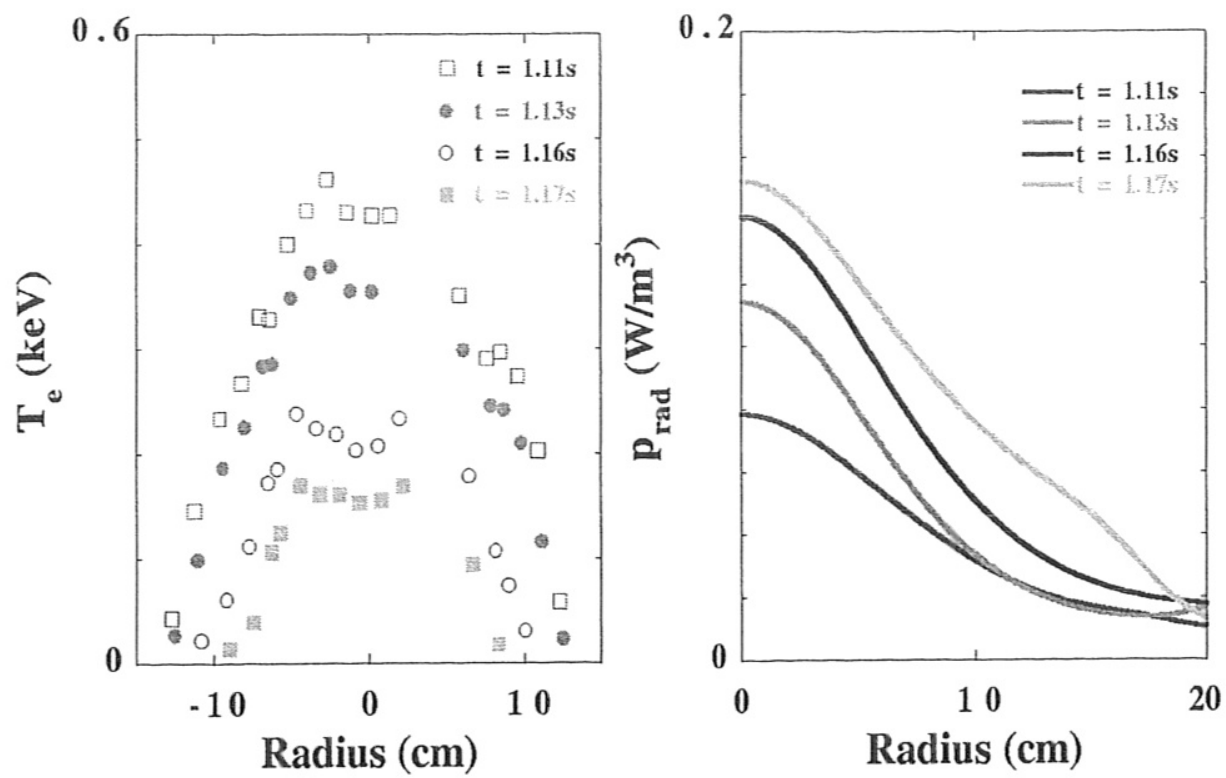

Figure 9: Evolution of electron temperature profiles from ECE and radiation profiles in the collapsing phase of the ramp down in ECRH power experiments. The rise in radiated power and fall in electron temperature in a time interval of $60 \mathrm{~ms}$ can be documented as ECE measurements are possible below the cut off density for the $140 \mathrm{GHz}$ gyrotrons. 

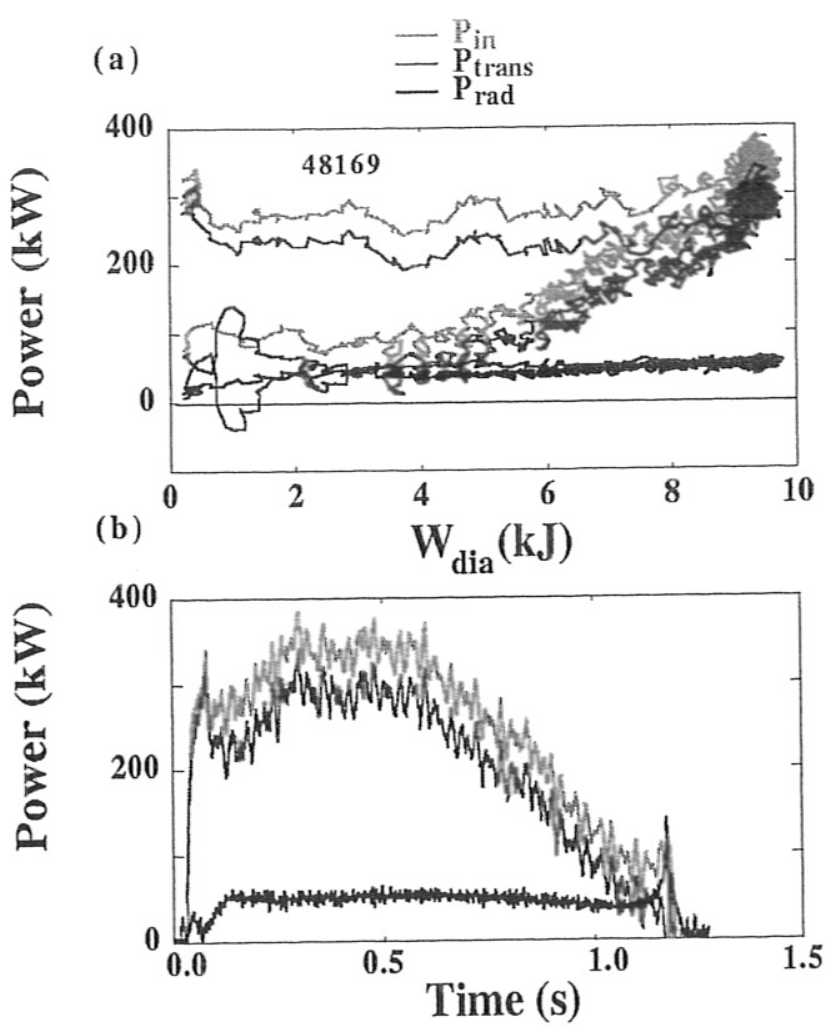

Figure 10: Evolution of total absorbed power, $\mathrm{P}_{a b s}$, radiated power, $\mathrm{P}_{\text {rad }}$ and the difference of these interpreted as the power carried by transport processes, $\mathrm{P}_{\text {trans }}$, plotted as (a) a function of diamagnetic energy or (b) time. At the density limit the radiated power approaches the absorbed power. 
-48169 B=2.5 T P $\mathrm{P}_{\mathrm{ECRH}}=0.5 \mathrm{MW}+$ ramp down

$-48077 \mathrm{~B}=1.25 \mathrm{~T} \mathrm{P}_{\mathrm{NBI}}=0.5 \mathrm{MW}$

$-48078 \quad \mathrm{~B}=1.25 \mathrm{~T} \mathrm{P}_{\mathrm{NB1}}=1.0 \mathrm{MW}$

$-48064 B=2.5$ T P $P_{\mathrm{NB1}}=0.5 \mathrm{MW}$

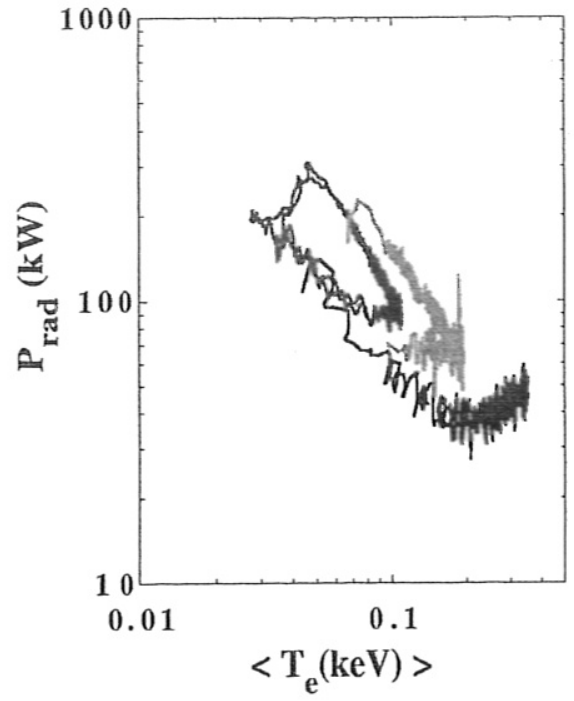

$-48067 \quad \mathrm{~B}=2.50 \mathrm{~T} \mathrm{P}_{\mathrm{NBI}}=1.0 \mathrm{WW}$

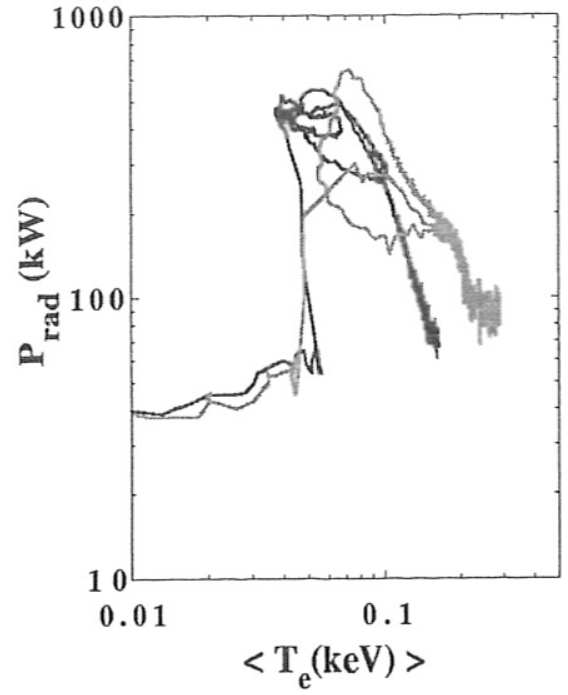

Figure 11: Comparison of radiated power versus derived average temperature at magnetic fields of $1.25 \mathrm{~T}$ and $2.55 \mathrm{~T}$ with a) $\mathrm{P}_{N B I}=0.5 \mathrm{MW}$ or $\mathrm{P}_{E C R H}=0.5 \mathrm{MW}$ and b) $\mathrm{P}_{N B I}=1.0 \mathrm{MW}$ at a rotational transform, $\iota$, of 0.34 . The observed hysteresis is caused by a density collapse at the density limit. 


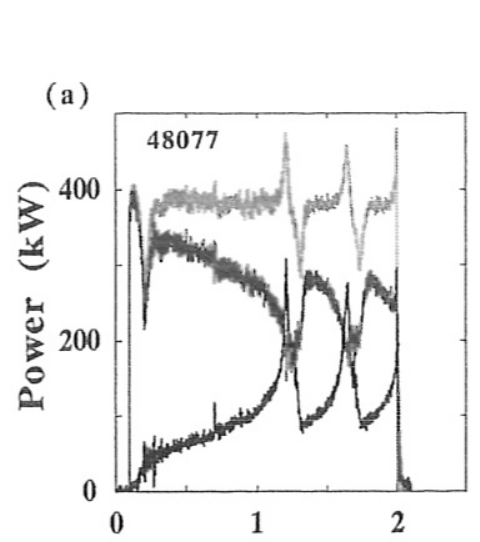

- $\mathrm{P}_{\text {in }}$
- $\mathrm{P}_{\text {rad }}$
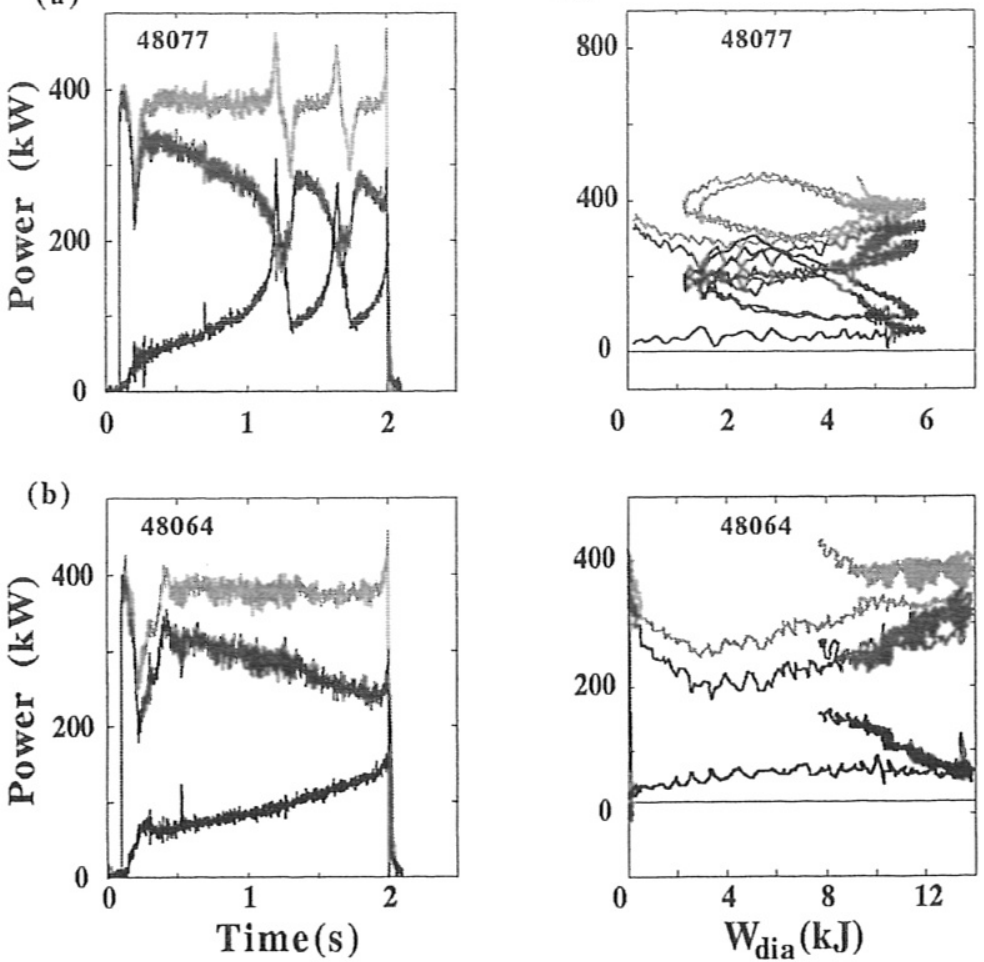

Figure 12: Evolution of total absorbed power, $\mathrm{P}_{a b s}$, radiated power, $\mathrm{P}_{\text {rad }}$ and the difference of these interpreted as the power carried by transport processes, $\mathrm{P}_{\text {trans }}$, for the $\mathrm{P}_{N B I}=0.5 \mathrm{MW}$ discharges at (a) $\mathrm{B}=1.25 \mathrm{~T}$ and (b) $\mathrm{B}=2.5 \mathrm{~T}$ plotted as a function of time (left column) or diamagnetic energy (right column). The hysteresis nature of $\mathrm{P}_{\text {rad }}(\mathrm{W})$ is contrasted to $\mathrm{P}_{\text {trans }}(\mathrm{W})$ which within errors is a single valued function during the cycle of diamagnetic energy. 

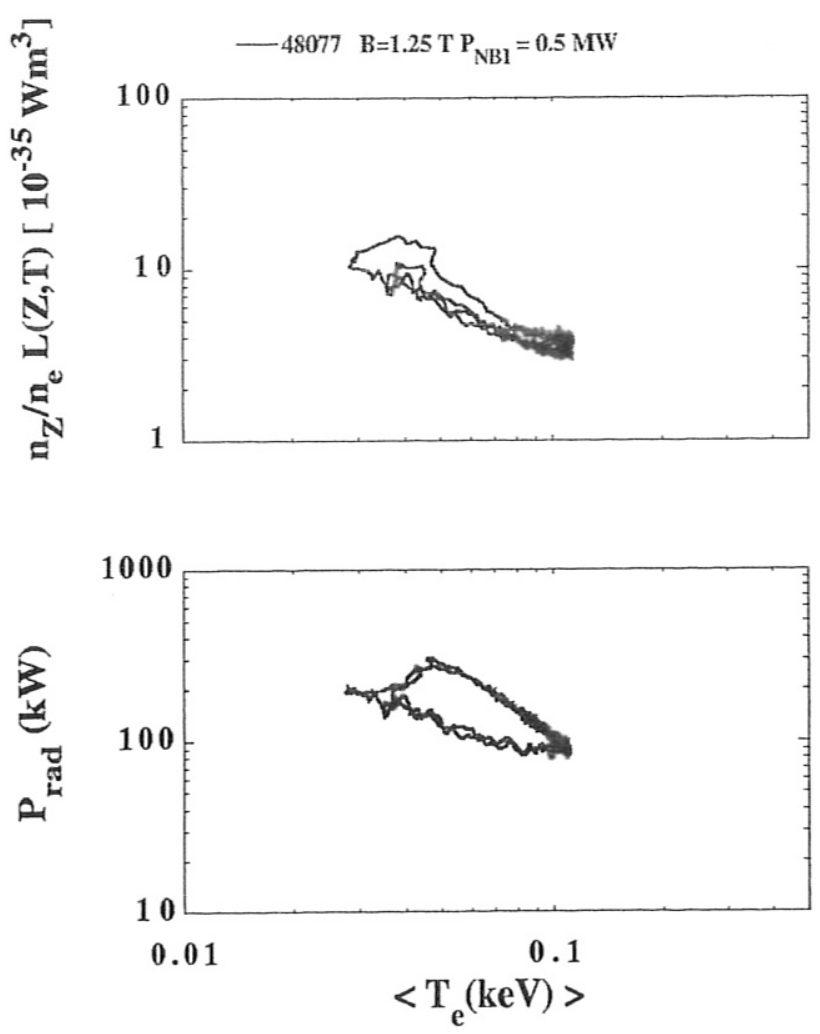

Figure 13: Total radiated power and the normalized radiated power of Eqn. 21 for the $\mathrm{P}_{N B I}=0.5 \mathrm{MW}$ discharge at $\mathrm{B}=1.25 \mathrm{~T}$ plotted as a function of derived mean temperature. The observed hysteresis in radiated power is therefore due to the density collapse at the density limit. 

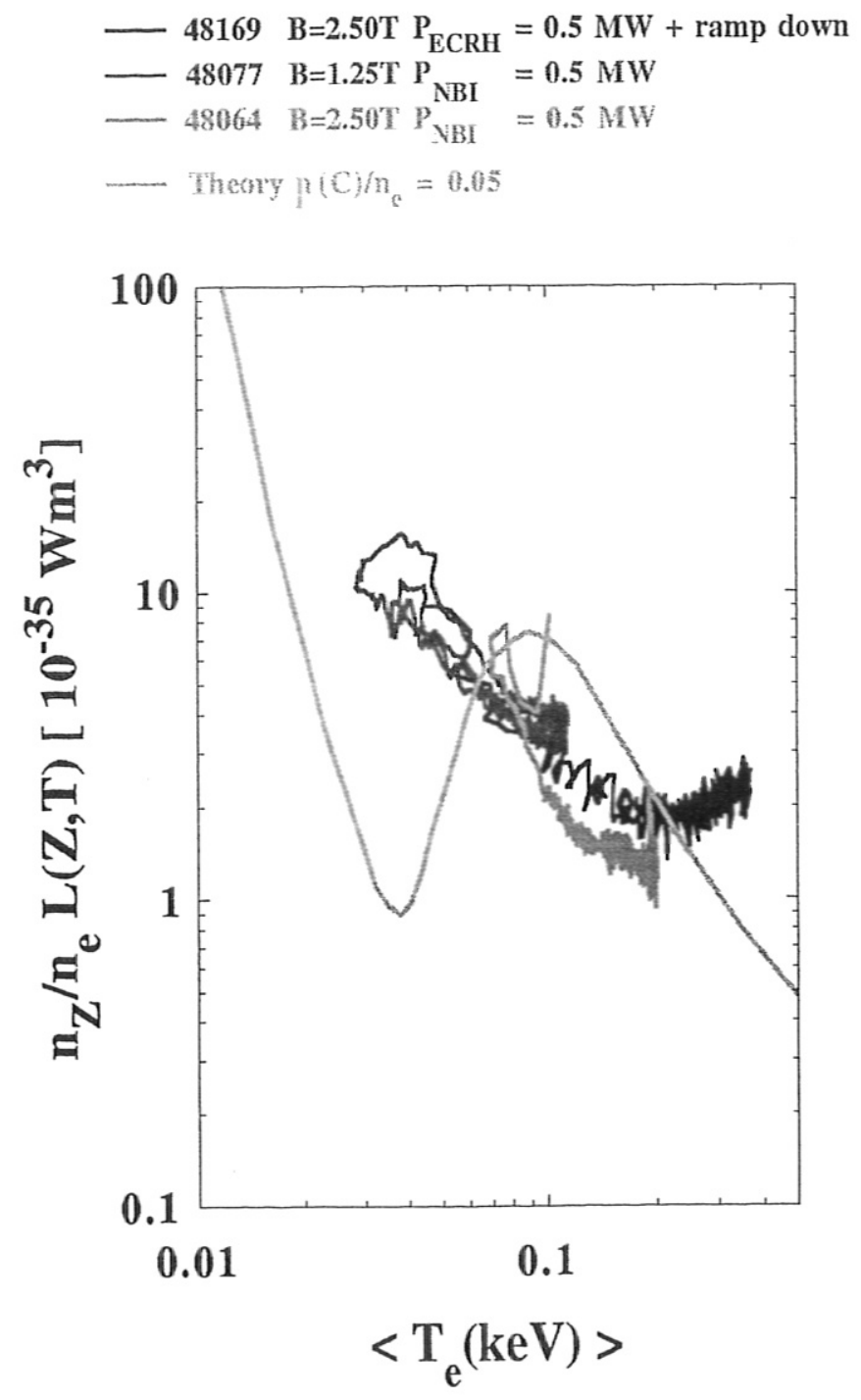

Figure 14: Comparison of experimental cooling curves in discharges with $\mathrm{P}_{N B I}$ or $\mathrm{P}_{E C R H}=0.5 \mathrm{MW}$ at a rotational transform, $\iota$, of 0.34 . For comparison the cooling curve of carbon with $5 \%$ relative concentration is shown. The presentation of the measurements in this form allows a simple but rapid comparison of the impurity content of different discharges. 

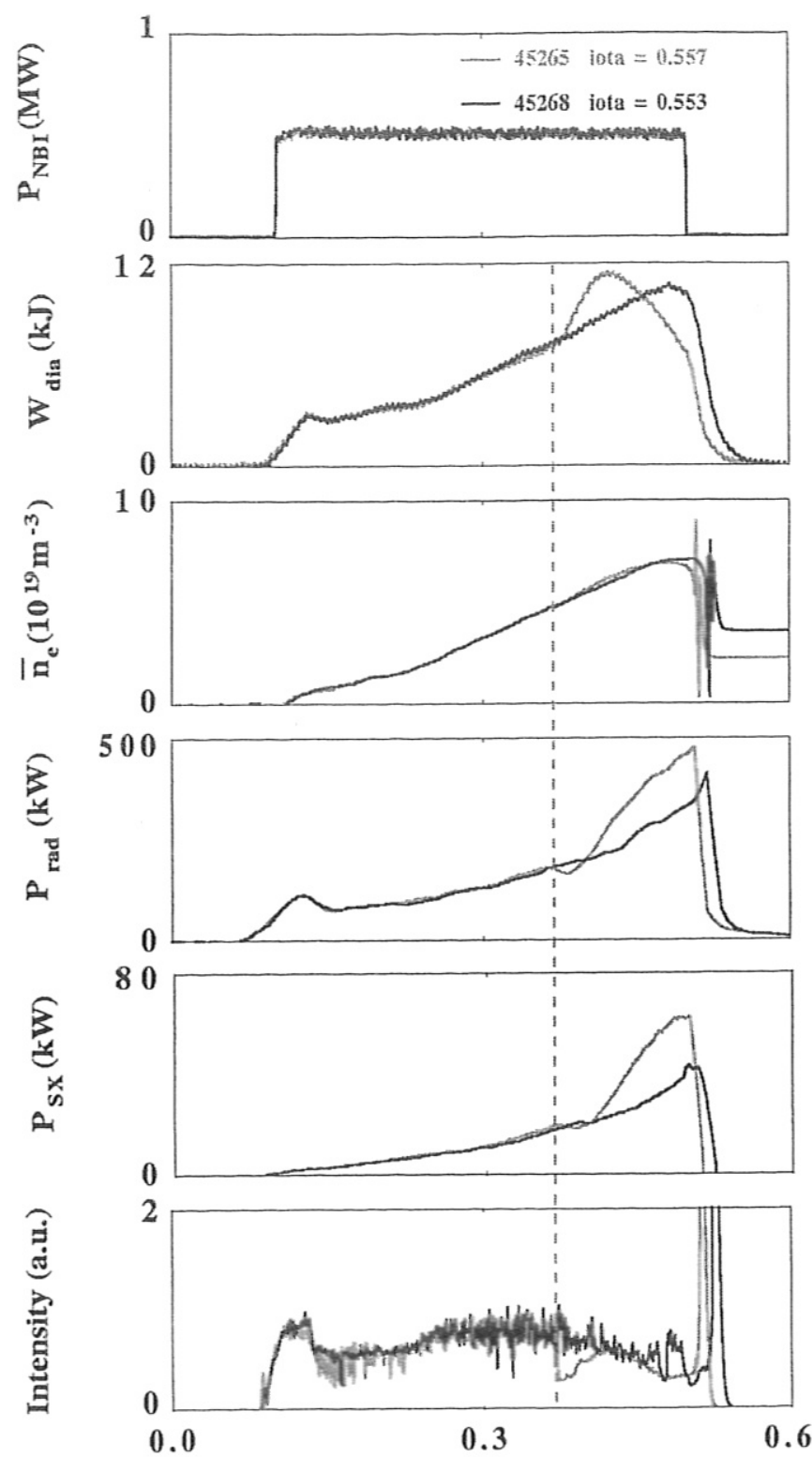

Time(s)

Figure 15: Comparison of evolution of radiated power in discharges in the vicinity of a rotational transform, $\iota$, of 0.56 with $\mathrm{B}_{o}=2.5 \mathrm{~T}$ and $\mathrm{P}_{N B I}=0.5 \mathrm{MW}$. The H-mode transition at the time given by the dotted line is indicated by the fall in the $\mathrm{H}_{\alpha}$ trace. 

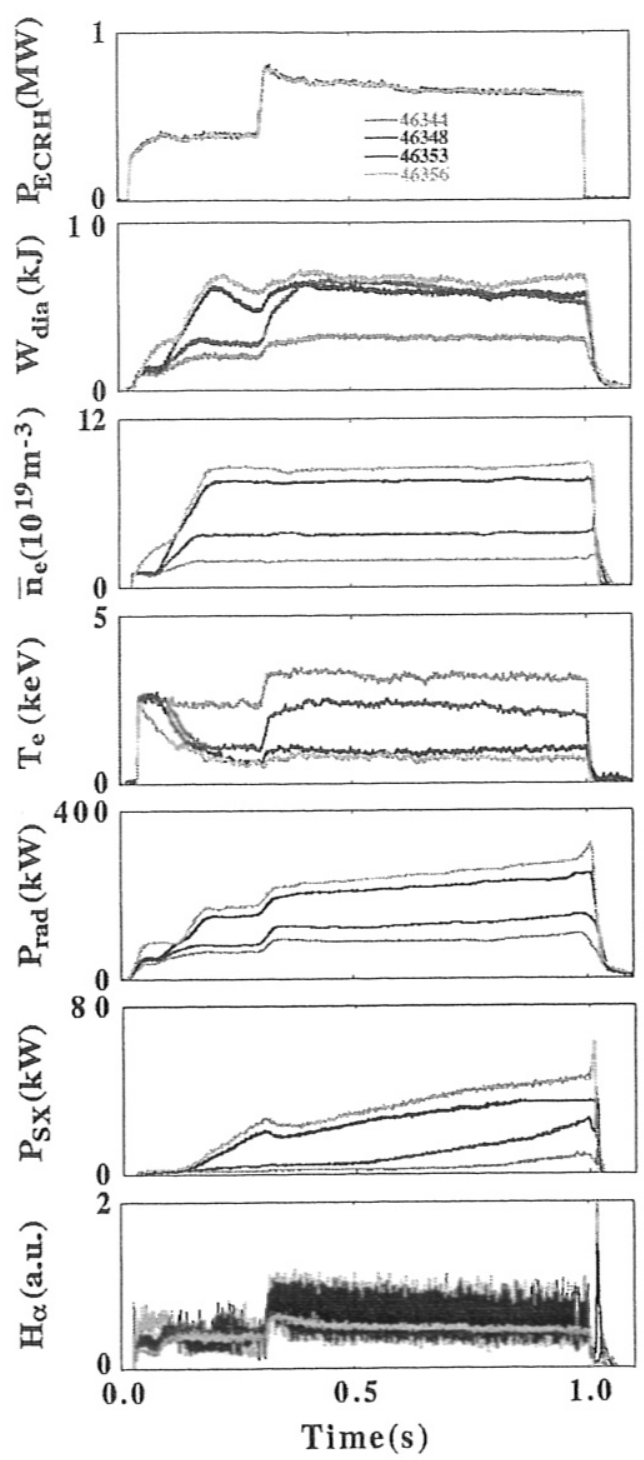

Figure 16: Overview of a density scan in ECRH discharges at $\mathrm{B}_{o}=2.5 \mathrm{~T}$ and $\iota(\mathrm{a})=0.564$ with $\mathrm{P}_{E C R H}=1.0 \mathrm{MW}$. Only the discharges at the two highest densities are in the H-mode. As indicated by the $\mathrm{H}_{\alpha}$ traces these discharges have considerably less ELM activity than those discharges at the lower density. 


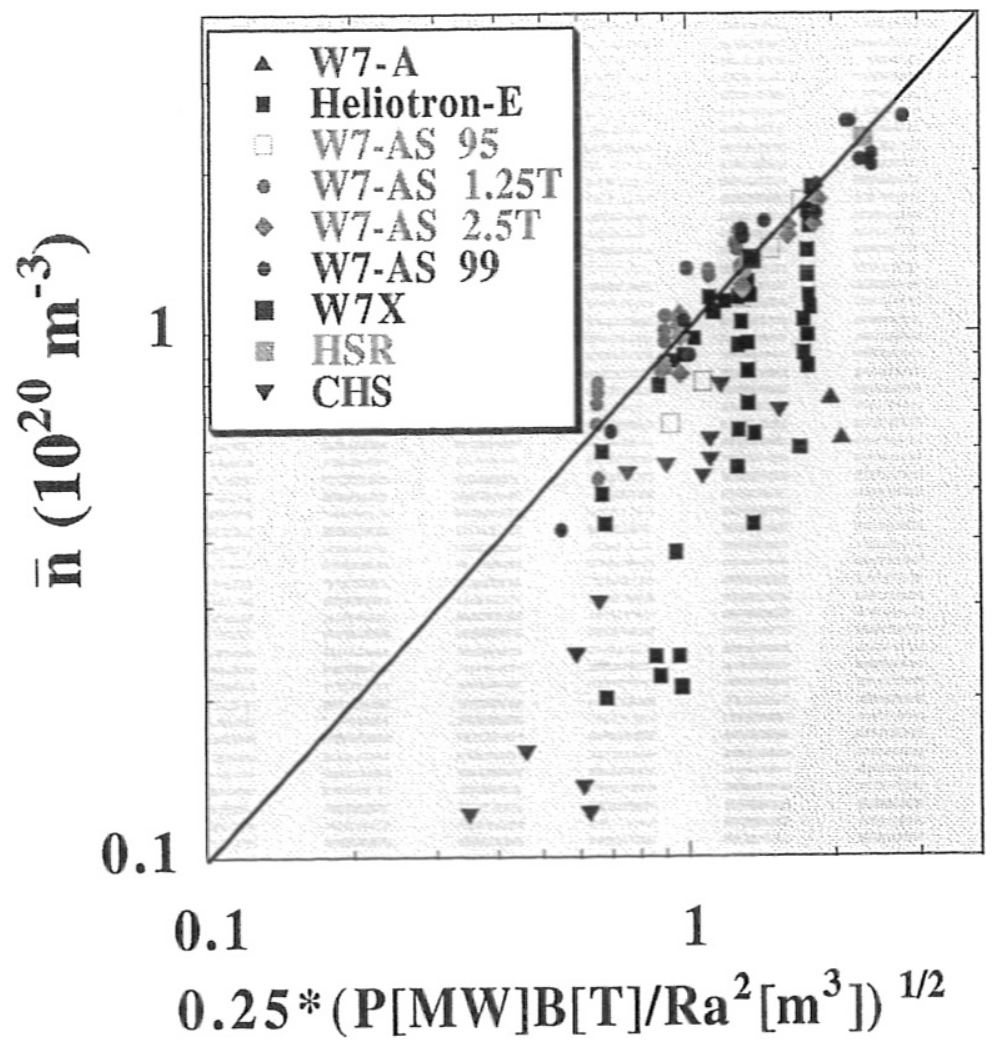

Figure 17: Comparison of empirical stellarator scaling law (SUDO et al., 1990) for density limit discharges using W7-A, Heliotron-E and W7-AS discharges. 